\title{
Investigation of the Casimir interaction between two magnetic metals in comparison with nonmagnetic test bodies
}

\author{
A. A. Banishev* ${ }^{1}$ G. L. Klimchitskaya,${ }^{2}$ V. M. Mostepanenko, ${ }^{2}$ and U. Mohideen ${ }^{1}$ \\ ${ }^{1}$ Department of Physics and Astronomy, \\ University of California, Riverside, California 92521, USA \\ ${ }^{2}$ Central Astronomical Observatory at Pulkovo of the \\ Russian Academy of Sciences, St.Petersburg, 196140, Russia
}

\begin{abstract}
We present the complete results for the dynamic experiment on measuring the gradient of the Casimir force between magnetic (Ni-coated) surfaces of a plate and a sphere. Special attention is paid to the description of some details of the setup, its calibration, error analysis and background effects. Computations are performed in the framework of the Lifshitz theory at nonzero temperature with account of analytic corrections to the proximity force approximation and of surface roughness using both the Drude and the plasma model approaches. The theory of magnetic interaction between a sphere and a plate due to domain structure of their surfaces is developed for both out-of-plane and in-plane magnetizations in the absence and in the presence of spontaneous magnetization. It is shown that in all cases the magnetic contribution to the measured force gradients is much smaller than the total experimental error. The comparison between experiment and theory is done using the rigorous statistical method. It is shown that the theoretical approach taking into account dissipation of free electrons is excluded by the data at a $95 \%$ confidence level. The approach neglecting dissipation is confirmed by the data at more than $90 \%$ confidence level. We prove that the results of experiments with $\mathrm{Ni}-\mathrm{Ni}, \mathrm{Ni}-\mathrm{Au}$ and $\mathrm{Au}-\mathrm{Au}$ surfaces taken together cannot be reconciled with the approach including free electrons dissipation by the introduction of any unaccounted background force, either attractive or repulsive.
\end{abstract}

PACS numbers: 78.20.Ls, 12.20.Fv, 75.50.-y, 78.67.Bf

\footnotetext{
* Present adress: Department of Chemistry, University of Illinois, Urbana, Illinois 61801, USA
} 


\section{INTRODUCTION}

The Casimir interaction is a version of the van der Waals interaction ${ }^{1}$ when the separation distance between the interacting bodies exceeds a few nanometers, and relativistic effects make an important contribution. The investigation of this phenomenon goes back to the seminal paper by Casimir ${ }^{2}$ which predicted that there is an attractive force between two neutral parallel ideal metal plates in vacuum. The Casimir force originates from the existence of zero-point oscillations of the electromagnetic field and thermal photons. Lifshitz ${ }^{3}$ developed the general theory of the van der Waals and Casimir forces between plates made of different materials based on the theory of electromagnetic fluctuations. At the present time the Casimir effect is investigated along with other quantum phenomena caused by fluctuat-

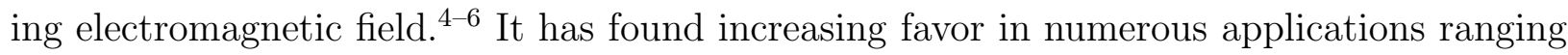
from condensed matter physics, atomic physics to elementary particle physics, astrophysics and cosmology $\underline{\underline{7}}^{\underline{-9}}$ Much attention is given to measurements of the Casimir force between two test bodies made of different materials. Thanks to modern laboratory techniques using atomic force microscopes (AFM) and micromachined oscillators it has been made possible to measure the Casimir interaction to a high precision at submicrometer separation distances (see reviews in Refs. $\stackrel{10}{12}^{-1}$ ). In comparisons between experiment and theory, some unexpected features in the interaction of quantum fluctuations with matter have been found connected with the role of conduction electrons which remain poorly understood up to the present (see below in Secs. VI and VII).

The original version of the Lifshitz theory ${ }^{\frac{3}{3}}$ describes materials of the test bodies by means of a single quantity, the frequency-dependent dielectric permittivity $\varepsilon(\omega)$. In so doing the main physical observables, such as the Casimir free energy and force, are most conveniently expressed via $\varepsilon\left(i \xi_{l}\right)$ where the Matsubara frequencies are $\xi_{l}=2 \pi k_{B} T l / \hbar, k_{B}$ is the Boltzmann constant, $T$ is the temperature, $l=0,1,2, \ldots$, and $\hbar$ is the Planck constant. The magnetic permeability of materials was assumed to be equal to unity, $\mu\left(i \xi_{l}\right)=1$. This is justified for diamagnets whose magnetic properties are characterized by the relation $\underline{13}-15$ $|\mu(0)-1| \sim 10^{-5}$. For paramagnets consisting of paramagnetic magnetizable microparticles with no intrinsic magnetic moment (the Van Vleck polarization paramagnetism ${ }^{16}$ ) the magnetic properties are also negligibly small. The same holds for paramagnets in the narrow sense which consist of microparticles possessing an intrinsic (permanent) magnetic moments 
whose interaction remains negligibly small even with the decrease of temperature to absolute zero $\stackrel{13}{-16}^{-16}$ This allows one to conclude $\frac{17}{}$ that in most of cases the contribution of the magnetic properties to the Casimir interaction is very small. There is, however, the subset of paramagnets in the broad sense called ferromagnets whose atoms possess strongly interacting constituent magnetic moments below the temperature of the magnetic phase transition (the Curie temperature $T_{C}$ ). This results in large magnetic permeabilities at zero Matsubara frequency, $\mu(0) \gg 1$, in the temperature region $T<T_{C}$. Richmond and Ninham $\frac{18}{}$ have generalized the Lifshitz theory for the case of interacting bodies described by the dielectric permittivity $\varepsilon\left(i \xi_{l}\right)$ and magnetic permeability $\mu\left(i \xi_{l}\right)$ calculated at imaginary Matsubara frequencies.

After generalization of the Lifshitz theory for the case of magnetic plates, much theoretical work has been done. Specifically, all main equations of the theory were obtained 19,20 for an arbitrary number of plane parallel layers of magnetodielectrics possessing different $\varepsilon\left(i \xi_{l}\right)$ and $\mu\left(i \xi_{l}\right)$. Furthermore, the Lifshitz theory of van der Waals and Casimir interactions was formulated for magnetodielectric bodies of arbitrary shape. ${ }^{21}$ Many papers aimed to use magnetic properties in order to realize the Casimir repulsion. ${ }^{22-29}$ It was understood, 25,26 however, that $\mu\left(i \xi_{l}\right)$ decreases rapidly with $l$ in accordance with the Debye formula 15

$$
\mu\left(i \xi_{l}\right)=1+\frac{\mu(0)-1}{1+\xi_{l} / \omega_{m}}
$$

where $\omega_{m}$ is the characteristic frequency which is much less than $\xi_{1} \sim 10^{14} \mathrm{~Hz}$ at room temperature. For ferromagnetic metals $\mu(i \xi)$ becomes equal to unity at $\xi>10^{5} \mathrm{~Hz}$ (see, e.g., Ref. $\left.{ }^{30}\right)$. From this it follows that the magnetic Casimir interaction is determined by only the zero-frequency Matsubara term (i.e., the term with $l=0$ in the Lifshitz formula). As a result, under some conditions the magnetic repulsion is now expected only between two test bodies one of which is made of ferromagnetic dielectric and another of a nonmagnetic metal. $\underline{24}-\underline{26}$ In parallel with the magnetic Casimir interaction between two macroscopic bodies the case of polarizable microparticles (atoms) with both electric and magnetic polarizabilities was considered. $\frac{29}{}$ It was found that magnetic properies of both atoms and material of the wall influence the atom-wall interaction. 19,31,32

Recent Ref. .33 marked the beginning of experimental research of the magnetic Casimir interaction. In this experiment the dynamic AFM operated in the frequency-shift mode was used to measure the gradient of the Casimir force between an Au-coated sphere of $64.1 \mu \mathrm{m}$ 
radius oscillating in perpendicular direction to the plate covered with the ferromagnetic metal Ni. The dymanic AFM technique with a sharp tip has been used for mapping surface topography for many years. ${ }^{34}$ For measurements of the gradient of the Casimir force the dynamic AFM was used in the phase-shift $\underline{35,36}$ and in the amplitude-shift $\frac{37,38}{38}$ modes. When using the dynamic AFM in the frequency-shift mode, the gradient of the Casimir force acting on the cantilever modifies the resonant frequency and the corresponding frequency shift is measured by means of a phase locked loop (PLL). For AFM with a sharp tip this measurement mode was discussed in detail in Ref 39 . To measure the Casimir interaction by means of an AFM, it was originally applied $\underline{40}$ in the configuration of an Au-coated sphere oscillating near an Au-coated plate. Previously dynamic measurements of the Casimir interaction in

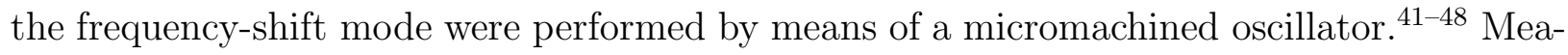
surements of the Casimir interaction between an Au-coated sphere and a Ni-coated plate ${ }^{33}$ demonstrated the impact of magnetic properties of $\mathrm{Ni}$, as predicted by the Lifshitz theory with neglected relaxation properties of conduction electrons (this theoretical approach was experimentally confirmed previously by measurements with two Au test bodies; $\stackrel{40,43-46}{46}$ see Secs. VI and VII for a complete discussion). However, with inclusion of the relaxation properties of free charge carriers, the Lifshitz theory does not predict any impact of magnetic properties on the Casimir interaction in the Au-Ni configuration. Unfortunately, both theoretical predictions, by coincidence, numerically almost overlap over the experimental separation region. This does not allow to conclude that Ref $\frac{33}{3}$ alone contains an independent confirmation for the impact of magnetic properties on the Casimir interaction.

The convincing confirmation for the role of magnetic properties in the Casimir effect was achieved ${ }^{49}$ by measuring the gradient of the Casimir force between a Ni-coated sphere and a Ni-coated plate by means of dynamic AFM operated in the frequency-shift mode. In this configuration the Lifshitz theory predicts sufficiently different values of the gradient of the Casimir force in cases when the relaxation properties of conduction electrons are either included or neglected, and in both cases the magnetic properties have a pronounced effect on the result. Using the same setup, as in Refs. $\frac{33}{}$ and $\stackrel{40}{ }$ for $\mathrm{Au}-\mathrm{Ni}$ and $\mathrm{Au}-\mathrm{Au}$ configurations, respectively, it was shown that the magnetic properties of $\mathrm{Ni}$ affect the measured gradient of the Casimir force. The experimental results were found to be in excellent agreement with the predictions of the Lifshitz theory with the relaxation properties of free charge carriers neglected. The theoretical predictions which take into account relaxation properties of free 
electrons were experimentally excluded at a high confidence level. ${ }^{49}$ Remarkably, for $\mathrm{Ni}-\mathrm{Ni}$ configuration the predictions of two theoretical approaches change places, as compared to the case of $\mathrm{Au}-\mathrm{Au}$ test bodies. ${ }^{49}$ This leads to important conclusions concerning the role of some possible background effects (see Secs. VI and VII).

The present paper contains full description of the experiment on measuring the gradient of the Casimir force between Ni-coated surfaces of a sphere and a plate which was briefly described in Ref. $\stackrel{49}{ }$. After a necessary short discussion about the measurement scheme (note that the setup is common for the experiments of Refs $\cdot \underline{33}, \underline{40}$ and ${ }^{49}$ ), we present the measurement results including those which were not published so far. The error analysis is elucidated in more detail including the random, systematic and total experimental errors. The Casimir interaction between two Ni-coated surfaces used in this experiment is calculated with the help of the Lifshitz theory within the two theoretical approaches either neglecting or taking into account the relaxation properties of free electrons. In so doing the corrections due to surface roughness and due to deviations from the proximity force approximation (PFA) are taken into account. Next, the detailed estimate of the magnetic interaction, which might act in the experimental setup due to the domain structure of the films independent of the Casimir interaction, is given. We demonstrate that the gradient of the magnetic force is sufficiently small and cannot interfere in the comparison between experiment and theory for both cases of magnetization perpendicular or parallel to the plane of the film. Then the obtained experimental results are compared with the results of numerical computations using the two theoretical approaches. This is done with the help of a more rigorous statistical method which was not used in Refs. ${ }^{33,40}$ and $\stackrel{49}{ }$. We arrive at the conclusion that the Lifshitz theory with omitted relaxation properties of free electrons is consistent with the measurement data whereas the same theory with the inclusion of relaxation properties is excluded by the data at a $95 \%$ confidence level. At the end of the paper we compare the experimental results of this experiment with experiments of Refs. $\underline{33}$ and $\underline{40}$ involving at least one nonmagnetic $(\mathrm{Au})$ surface.

The structure of the paper is as follows. In Sec. II we briefly present the measurement scheme using the dynamic AFM operated in the frequency-shift mode. Section III contains our measurement results and the analysis of errors. In Sec. IV the computational results for the gradient of the Casimir force between two Ni surfaces are presented. The calculation for the upper bound of the magnetic interaction in our setup can be found in Sec. V. In 
Sec. VI the reader will find the comparison between experiment and theory for two magnetic test bodies. Section VII contains the comparison with previously performed experiments. Section VIII is devoted to our conclusions and discussion. Appendices A and B contain some details of mathematical calculations.

\section{MEASUREMENT SCHEME USING DYNAMIC AFM}

The dynamic AFM operated in the frequency-shift mode, used in this experiment to measure the gradient of the Casimir force between Ni-coated surfaces of a hollow glass sphere of $R=61.71 \pm 0.09 \mu \mathrm{m}$ radius and a Si plate, is already described in Refs $\underline{33,40}$ and $\underline{\underline{49}}$. Here we present only a few main points necessary for understanding of the subsequent text and dwell only on details which were not discussed previously. The sphere was attached to the rectangular $\mathrm{Si}$ cantilever of an AFM and the plate was mounted on top of a piezoelectric tube capable of travelling a separation distance $z_{\text {piezo }}$ of $2.3 \mu \mathrm{m}$ between the surfaces of a sphere and a plate. The movement of the piezo was calibrated by a fiber interferometer. Both test bodies were cleaned using the special multi-step cleaning procedure and placed in the vacuum chamber that was capable of reaching a pressure of $10^{-9}$ Torr by using mechanical, turbo and ion pumps [see Fig. 1(a,b) in Ref. $\underline{\underline{40}}$ for a layout of the setup]. The piezoelectric tube contained a small magnet introduced by the piezotube manufacturer which is not needed in this experiment. The initial magnetic field was measured to be $\approx 100$ Gs using a Hall probe gaussmeter. To prevent any effects from this field, we inserted a piece of mu-metal magnetic shield between the top of the piezo tube and the Ni-coated plate. The residual magnetic field was below the detection resolution of $0.1 \mathrm{Gs}$. Both the initial and residual fields do not depend on separation in the separation region considered and do not contribute to the force gradient measured in our work.

In a dynamic experiment using the frequency-shift mode the measured quantity is the change of resonant frequency $\omega_{0}$ of the periodically driven cantilever. $\underline{34}$ The change of the resonant frequency from $\omega_{0}$ to $\omega_{r}$ occurs under the influence of an external force

$$
F_{\text {tot }}(a, T)=F_{\text {el }}(a)+F(a, T),
$$

acting between the sphere and the plate at the laboratory temperature $T=300 \mathrm{~K}$. Here $F_{\mathrm{el}}(a)$ is the electric force caused by the voltages $V_{i}$ applied to the plate whereas the sphere 
remains grounded and $F(a, T)$ is the Casimir force. The absolute separation between the sphere and plate surface is given by

$$
a=z_{\text {piezo }}+z_{0}
$$

where $z_{0}$ is the point of the closest approach between the two surfaces, which is much larger than the separation on contact in the dynamic experiments. Note that even if $V_{i}=0$ there is some residual potential difference $V_{0}$ between the sphere and the plate caused by different connections and work functions of the polycrystalline surface from patches and possible adsorbates on their surfaces.

The shift of the resonance frequency of the cantilever was detected by means of an optical interferometer ${ }^{50,51}$ To prevent any error in the sphere-plate separation $a$ due to cantilever deflection under the influence of a force $F_{\text {tot }}(a, T)$, we have kept the interferometric cavity length constant by means of an additional piezo, which was controlled by a proportionalintegral-derivative feedback loop. Then the frequency shift

$$
\Delta \omega(a)=\omega_{r}(a)-\omega_{0},
$$

was measured by the PLL frequency demodulator system (here and below we omit an argument $T$ in the frequency shift because $T$ is kept constant). The output of the feedback loop provided by the PLL was the resonant-frequency shift $\omega_{r}(a)-\omega_{d}$, where $\omega_{d}$ is the set-point frequency of the PLL. We made sure that at large separations above $2.2 \mu \mathrm{m}$ the frequency shift $\omega_{r}(a)-\omega_{d}$ remains constant within the resolution limit. From this it follows that at separations above $2.2 \mu \mathrm{m}$ there is no influence of the external force and $\omega_{r}(a)=\omega_{0}$. Finally the frequency shift (44) was found from the two measured quantities by the equation

$$
\Delta \omega(a)=\left[\omega_{r}(a)-\omega_{d}\right]-\left(\omega_{0}-\omega_{d}\right)
$$

In the linear regime, which holds for sufficiently small oscillation amplitudes of the cantilever, the frequency shift is given by $\underline{\underline{40}}$

$$
\Delta \omega(a)=-\frac{\omega_{0}}{2 k} \frac{\partial F_{\text {tot }}(a, T)}{\partial a} \equiv-\frac{\omega_{0}}{2 k} F_{\text {tot }}^{\prime}(a, T)
$$

where $k$ is the spring constant of the cantilever (maximum allowed amplitudes ensuring the applicability of the linear regime are calculated in Ref. $\stackrel{40}{ })$. 
The electric force contributing to the total force (2) in the configuration of a metal sphere above a metal plate can be calculated precisely as $\frac{9.52}{\underline{.52}}$

$$
F_{\mathrm{el}}(a)=X(a, R)\left(V_{i}-V_{0}\right)^{2} .
$$

Here the function $X(a, R)$ is given by

$$
\begin{aligned}
& X(a, R)=2 \pi \epsilon_{0} \sum_{n=1}^{\infty} \frac{\operatorname{coth} \alpha-n \operatorname{coth}(n \alpha)}{\sinh (n \alpha)}, \\
& \cosh \alpha=1+\frac{a}{R},
\end{aligned}
$$

where $\epsilon_{0}$ is the permittivity of the vacuum. When using Eq. (8) in electrostatic calibrations (see Sec. III), it is convenient to present $X(a, R)$ as the sum of powers 9.53

$$
X(a, R)=-2 \pi \epsilon_{0}\left[c_{-1} \frac{R}{a}+c_{0}+c_{1} \frac{a}{R}+c_{2} \frac{a^{2}}{R^{2}}+\ldots\right],
$$

where $c_{1}=0.5, c_{0}=-1.18260, c_{1}=22.2375, c_{2}=-571.366$ etc. Substituting Eqs. (2) and (77) in Eq. (6), one can connect the measured frequency shift with the gradient of the Casimir force

$$
\Delta \omega(a)=-\beta\left(V_{i}-V_{0}\right)^{2}-C \frac{\partial F(a, T)}{\partial a} .
$$

Here $C \equiv \omega_{0} /(2 k)$ and $\beta \equiv \beta\left(z_{0}, z_{\text {piezo }}, C, R\right)=C \partial X(a, R) / \partial a$. Substituting Eq. (9) in the definition of $\beta$, one obtains

$$
\beta=\frac{\pi \epsilon_{0} R C}{a^{2}}\left(1-2 c_{1} \frac{a^{2}}{R^{2}}-4 c_{2} \frac{a^{3}}{R^{3}}+\ldots\right),
$$

where $a$ is expressed according to Eq. (3).

\section{MEASUREMENT RESULTS AND ERROR ANALYSIS FOR TWO MAGNETIC BODIES}

To find the gradient of the Casimir force as a function of separation from the measured frequency shift by using Eq. (10), one needs sufficiently precise values of the coefficients $C$ and $\beta$, of the residual potential difference $V_{0}$, and of the separation at the closest approach $z_{0}$. These were found by means of electrostatic calibrations which were performed using the dependence of the frequency shift on the applied voltages in Eq. (10). For this purpose 11 different voltages, $-64.5,-54.7,-45.1,-35.3,-25.5,-17.7,-7.8,2.2,12.5,22.3$ and 
$31.6 \mathrm{mV}$ were sequentially applied to the plate while the sphere remained grounded. With each applied voltage the plate was moved towards the sphere starting at the maximum separation of $2.3 \mu \mathrm{m}$ and the frequency shift $\Delta \omega(a)$ was recorded at each $0.14 \mathrm{~nm}$. To move the plate towards the sphere, continuous triangular voltages at $0.01 \mathrm{~Hz}$ were applied to the piezoelectric tube. The small mechanical drift in the $z_{\text {piezo }}$ was measured to be $0.003 \mathrm{~nm} / \mathrm{s}$ and corrected using the procedure described in Refs. ${ }^{33}$ and $\underline{40}$. Measurement of the frequency shift $\Delta \omega(a)$ was repeated three times with each applied voltage $V_{i}$. This resulted in 33 measurement sets.

To perform the electrostatic calibration, the measured frequency shift with a step of $1 \mathrm{~nm}$ was found by interpolation. Then at every $1 \mathrm{~nm} \Delta \omega$ was plotted as a function of the applied voltage $V_{i}$ and the value of $V_{0}$ was identified as the position of the parabola maximum. $\underline{33,40}$ The obtained values of $V_{0}$ as a function of separation are plotted in Fig. 1 of Ref. $\underline{49}$ over the separation region from 220 to $1000 \mathrm{~nm}$. As can be seen in this figure, $V_{0}$ does not depend on separation indicating that the interacting regions of the surfaces are clean or the adsorbed impurities are randomly distributed with a submicrometer size scale and make only a negligible contribution to the total force. ${ }^{40}$ The mean value of $V_{0}$ was found to be $V_{0}=-17.7 \pm 1 \mathrm{mV}$ (here and below the errors are indicated at a $67 \%$ confidence level if another value is not stated explicitly).

Next, we determined the coefficient $C$ and the separation at the closest approach $z_{0}$ by fitting the data for the parabola curvature $\beta$ to the theoretical expression in Eq. (11). A least $\chi^{2}$ fitting procedure was used which was repeated by keeping the start points fixed at the closest separation $z_{0}$, while the end point $z_{\text {end }}$ measured from $z_{0}$ was varied from 150 to $1190 \mathrm{~nm}$. In Fig. 1(a) the obtained values of $C$ are seen to be almost independent on the end point indicating the absence of systematic errors from the calibration of $z_{\text {piezo }}$, mechanical drift etc. The obtained mean value is $C=52.4 \pm 0.16 \mathrm{kHz}$ m/N. In Fig. 1(b) the respective values of $z_{0}$ are presented as a function of $z_{\text {end }}$. They are also independent of $z_{\text {end }}$ in the limits of errors of the fitting procedure. The mean value is $z_{0}=221.1 \pm 0.4 \mathrm{~nm}$. Then the absolute separations $a$ between the sphere and the plate are obtained from Eq. (33). The error in the determination of the absolute separations, $\Delta a$, is also equal to $0.4 \mathrm{~nm}$ because the relative separations, $z_{\text {piezo }}$, are determined to a much higher precision. We emphasize that our calibration parameters, including absolute separations, are determined with significantly smaller errors than it is common for sharp tips. The reason is that we 
use a large perfectly shaped sphere made from the liquid phase instead of rough surfaces where geometry is not known precisely. Another specific feature of our experiment is that the theoretical electric force in the sphere-plate geometry is known exactly and the electric potential can be determined to a high precision.

We are now in a position to find the gradients of the Casimir force $F^{\prime}(a, T) \equiv \partial F(a, T) / \partial a$ from the measured frequency shifts by using Eq. (10). They are again found at each $0.14 \mathrm{~nm}$ and then interpolated in order to get 33 values of the force gradient at each nanometer of the absolute separation $a$ (starting from $223 \mathrm{~nm}$ ). We have checked the statistical properties of the Casimir force gradient data obtained in this way and made sure that they are characterized by a Gaussian distribution (see Sec. VII for more details). In Fig. 2 we plot as dots all 33 data points for $F^{\prime}(a)$ with a step of $5 \mathrm{~nm}$ starting from the first integer separation $223 \mathrm{~nm}$, where our measurements were performed. The solid line shows the mean values of the measured gradients of the Casimir force found from 33 measurements. In the inset the same information is presented over a more narrow separation region which gives the possibility to demonstrate all the data points with a step of $1 \mathrm{~nm}$.

In this experiment the mean gradients of the Casimir force are burdened by errors of two types, the random and the systematic. The total experimental error is obtained as a combination of these two taking into account their distribution laws (see Refs. $\underline{\underline{9}}$ and $\underline{10}$ for details). The random error $\Delta^{r} F^{\prime}(a)$ calculated from 33 repetitions at a $67 \%$ confidence level using the Student distribution [the Student coefficient $t_{(1+0.67) / 2}(32)=1$ ] as a function of separation is shown by the short-dashed line in Fig. 3. As can be seen in the figure, $\Delta^{r} F^{\prime}(a)$ does not depend on separation. The systematic error is determined by the instrumental noise including the background noise level, by the errors in calibration, and by the errors in the gradient of the subtracted electrostatic force. Taking into account that all these errors are characterised by Gaussian distributions, to obtain the total systematic error $\Delta^{s} F^{\prime}(a)$ they were combined in quadrature. The obtained values of $\Delta^{s} F^{\prime}(a)$ at a $67 \%$ confidence level, as a function of separation, are shown in Fig. 3 by the long-dashed line. The increase of $\Delta^{s} F^{\prime}(a)$ at shorter separations is caused by the errors in the subtracted electrostatic force. As can be seen in Fig. 3, the systematic error is from a factor of 6 to a factor of 4 larger than the random error, as is typical for precise experiments of metrological quality. The total experimental error $\Delta^{t} F^{\prime}(a)$ at a $67 \%$ confidence level is obtained in quadrature from the random and systematic errors. It is shown by the solid line in Fig. 3. One can see that 
the total experimental error at all separations is mostly determined by the systematic error.

\section{CALCULATION OF THE CASIMIR INTERACTION BETWEEN TWO Ni BODIES}

Here we calculate the gradient of the Casimir force in the experimental configuration of a Ni-coated sphere and a Ni-coated plate. Given the thicknesses of Ni coatings $\left(d_{1}=250 \pm 1 \mathrm{~nm}\right.$ and $d_{2}=210 \pm 1 \mathrm{~nm}$ on the plate and the sphere, respectively), one can consider them as a solid Ni ball near a Ni semispace. $\frac{9}{}$ Using the PFA, the gradient of the Casimir force is given by

$$
F_{\mathrm{PFA}}^{\prime}(a, T)=2 \pi R \mathcal{F}_{p p}^{\prime}(a, T),
$$

where $\mathcal{F}_{p p}(a, T)$ is the free energy of the Casimir interaction per unit area of two parallel Ni semispaces spaced $a$ nanometers apart in thermal equilibrium at temperature $T$. According to the Lifshitz theory, ${ }^{3,18} \mathcal{F}_{p p}(a, T)$ can be presented as the sum from $l=0$ to $l=\infty$ over the Matsubara frequencies $\xi_{l}$ (see Sec. I). Then the gradient of the Casimir force (12) takes the form 40,49

$$
F_{\mathrm{PFA}}^{\prime}(a, T)=2 k_{B} T R \sum_{l=0}^{\infty} \int_{0}^{\infty} q_{l} k_{\perp} d k_{\perp} \sum_{\alpha} \frac{r_{\alpha}^{2}}{e^{2 a q_{l}}-r_{\alpha}^{2}} .
$$

Here, $q_{l}^{2}=k_{\perp}^{2}+\xi_{l}^{2} / c^{2}, k_{\perp}$ is the projection of the wave vector on the plate, and the prime following the summation sign multiplies the term with $l=0$ by $1 / 2$. The index $\alpha$ takes the two values TM and TE and denotes the transverse magnetic and transverse electric polarizations of the electromagnetic field. The respective reflection coefficients $r_{\alpha}$ calculated along the imaginary frequency axis have the following explicit form:

$$
\begin{aligned}
& r_{\mathrm{TM}} \equiv r_{\mathrm{TM}}\left(i \xi_{l}, k_{\perp}\right)=\frac{\varepsilon\left(i \xi_{l}\right) q_{l}-k_{l}}{\varepsilon\left(i \xi_{l}\right) q_{l}+k_{l}} \\
& r_{\mathrm{TE}} \equiv r_{\mathrm{TE}}\left(i \xi_{l}, k_{\perp}\right)=\frac{\mu\left(i \xi_{l}\right) q_{l}-k_{l}}{\mu\left(i \xi_{l}\right) q_{l}+k_{l}} \\
& k_{l}=\left[k_{\perp}^{2}+\varepsilon\left(i \xi_{l}\right) \mu\left(i \xi_{l}\right) \frac{\xi_{l}^{2}}{c^{2}}\right]^{1 / 2} .
\end{aligned}
$$

The main properties of the magnetic permeability $\mu$ of a boundary material (Ni) calculated at the imaginary Matsubara frequencies are discussed in Sec. I.

To apply Eqs. (13) and (14) for the calculation of the Casimir interaction, one needs to have the values of $\varepsilon\left(i \xi_{l}\right)$ up to sufficiently large values of $l$ and $\mu(0)$ (see Sec. I). The 
dielectric permittivity at the imaginary Matsubara frequencies is obtained by means of the Kramers-Kronig relation from $\operatorname{Im} \varepsilon(\omega)=2 n_{1}(\omega) n_{2}(\omega)$, where $n_{1}(\omega)$ and $n_{2}(\omega)$ are the real and imaginary parts of the complex index of refraction, respectively, measured and tabulated over a wide frequency region. $\underline{\underline{54}}$ An application of the Kramers-Kronig relation requires, however, the optical data at much lower frequencies than it may become available in any foreseeable future. Because of this, the problem arises on how to extrapolate the data for $\operatorname{Im} \varepsilon(\omega)$ to lower frequencies down to zero frequency. In Sec. I two approaches to the resolution of this problem proposed in the literature are mentioned. According to the first approach, which seems to be the most natural and straightforward from a theoretical point of view, in any extrapolation the properties of boundary materials should be described as precise as possible. Specifically, the relaxation properties of conduction electrons at low frequencies should be taken into account by means of the commonly accepted Drude dielectric function

$$
\varepsilon_{D}(\omega)=1-\frac{\omega_{p}^{2}}{\omega[\omega+i \gamma(T)]},
$$

where $\omega_{p}$ is the plasma frequency and $\gamma(T)$ is the relaxation parameter. This approach was called the Drude model approach. The theoretical predictions for the Casimir interaction obtained in this way were excluded by several experiments with metallic test

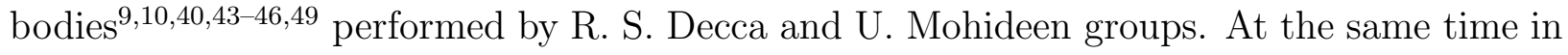
two other experiments 55,56 of S. K. Lamoreaux group the Drude model approach was claimed to be in agreement with the data. This conclusion, however, has been questioned $\underline{57} \underline{60}$ Furthermore, several experiments performed by E. A. Cornell and U. Mohideen groups with dielectric materials turned out to be in contradiction with theoretical predictions if the free charge carriers are included in the Lifshitz theory $\underline{9}, \underline{10}-66$ Besides, the inclusion of the relaxation properties of charge carriers or taking into account the free charge carriers for dielectrics in the Lifshitz theory were found to violate the third law of thermodynamics (the Nernst heat theorem) $\underline{6}, \underline{10,67}, 68$ This, however, happens at zero temperature and is not directly relevant to any experimental work.

The second proposed approach suggested to extrapolate the optical data for metals to zero frequency by means of the plasma model $\varepsilon_{p}(\omega)$. The latter is obtained from Eq. (15) by putting $\gamma(T)=0$, i.e., by disregarding the relaxation properties of free charge carriers (note that for permittivities having the second order pole at zero frequency the Kramers-Kronig relation is modified accordingly $\left.{ }^{69}\right)$. The plasma model approach was found to be consistent 
with the measurement data of the experiments with metallic test bodies. $\underline{9,10,40,43-46,49}$ The Drude and the plasma model approaches are the subject of continuing discussions in the literature $\stackrel{70-73}{ }$ Below we perform computations using both approaches on equal terms and compare the obtained results between themselves and with the measurement data.

We obtained the dielectric permittivity $\varepsilon\left(i \xi_{l}\right)$ from the optical data ${ }^{54}$ for the complex index of refraction of Ni using the Kramers-Kronig relation. The data were first extrapolated to zero frequency by using either the Drude or the plasma models. In so doing we have used the plasma frequency of $\mathrm{Ni} \omega_{p}=4.89 \mathrm{eV}$ and the relaxation parameter at $T=300 \mathrm{~K}$ $\gamma=0.0436 \mathrm{eV}$ according to Refs, $\underline{\underline{54}}$ and $\underline{74}$. Our Ni-coated test bodies did not possess a spontaneous magnetization due to sufficiently thick coatings and weak environment magnetic fields. The magnetic properties of $\mathrm{Ni}$ were described by a static magnetic permeability $\mu(0)=110$. For all Matsubara frequencies with $l \geq 1$ at $T=300 \mathrm{~K}$ it holds $\mu\left(i \xi_{l}\right)=1$ because $\mu(i \xi)$ rapidly falls to unity with increasing $\xi$ (see Sec. I).

Equation (13) was obtained using the PFA and, thus, is not exact. Recently the gradient of the Casimir force in a sphere-plate configuration was calculated exactly and the corrections to the PFA result were found $\underline{75}^{-78}$ According to these papers the exact force gradient between the sphere of large radius and the plate is equal to

$$
\begin{aligned}
F^{\prime}(a, T) & =F_{\mathrm{PFA}}^{\prime}(a, T)\left[1+\delta_{\mathrm{corr}}^{\mathrm{PFA}}(a, T, R)\right] \\
& =F_{\mathrm{PFA}}^{\prime}(a, T)\left[1+\theta(a, T) \frac{a}{R}+o\left(\frac{a}{R}\right)\right],
\end{aligned}
$$

where $F_{\mathrm{PFA}}^{\prime}$ is given in Eq. (13). In Ref $\underline{77}$ the coefficient $\theta$, as a function of separation, was calculated for Au at both $T=0$ and $T=300 \mathrm{~K}$ using the Drude model approach. In the separation region from 220 to $550 \mathrm{~nm}$ the obtained results at $T=300 \mathrm{~K}$ only slightly differ from those for ideal metal surfaces considered at $T=300 \mathrm{~K}$ in the framework of thermal quantum field theory. This demonstrates a very weak dependence of $\theta$ on the plasma frequency $\omega_{p}$, relaxation parameter $\gamma$ and optical data within this separation region. Because of this, one can use the values of $\theta$ found in Ref. $\stackrel{77}{\text { for }} \mathrm{Ni}$ as well. It was also shown $\underline{78}$ that at $T=0$ the values of $\theta$ calculated using the plasma model are sandwiched between those calculated using the Drude model and for ideal metal surfaces. This allows one to approximate the values of $\theta$ at $T=300 \mathrm{~K}$ in the plasma model approach by those for ideal metals at the same temperature. In Fig. 4 we present by the upper and lower solid lines the correction to PFA $\delta_{\text {corr }}$ at $T=300 \mathrm{~K}$ in percent for the Drude and plasma model approaches, 
respectively (note that the correction of order $a^{2} / R^{2} \sim 0.3 \times 10^{-4}$ can be neglected). As can be seen in Fig. 4, the error from using the PFA is substantially smaller than $a / R$. The latter value for this error was used previously. $10,40,43-46,49,61,62,65,66$ Thus, the analysis of previous experiments was highly conservative.

One more correction factor which should be introduced in Eq. (13) is due to the surface roughness. The root-mean-square roughness on the sphere and the plate was investigated by means of an AFM with a sharp tip and found to be $\delta_{s}=1.5 \mathrm{~nm}$ and $\delta_{p}=1.4 \mathrm{~nm}$, respectively. For so small $a$ roughness at separations above $200 \mathrm{~nm}$ one can use the multiplicative approach. $\underline{9,10}$ In the framework of this approach the force gradient with account of surface roughness is given by $\underline{9}, 10$

$$
\begin{aligned}
& F_{R}^{\prime}(a, T)=F^{\prime}(a, T)\left[1+\delta_{\mathrm{corr}}^{R}(a)\right], \\
& \delta_{\mathrm{corr}}^{R}(a)=10 \frac{\delta_{s}^{2}+\delta_{p}^{2}}{a^{2}}+105 \frac{\left(\delta_{s}^{2}+\delta_{p}^{2}\right)^{2}}{a^{4}} .
\end{aligned}
$$

In Fig. 4 the correction due to surface roughness $\delta_{\text {corr }}^{R}$ in percent is shown by the dashed line as a function of separation. As can be seen in Fig. 4, the corrections due to deviations from the PFA and due to surface roughness are of opposite signs and give only minor contributions to the force gradient.

Now we are in a position to calculate the gradient of the Casimir force $F^{\prime}(a, T)$ between two Ni surfaces at $T=300 \mathrm{~K}$ with account of all correction factors. Computations were performed by Eqs. (13), (14), (16), and (17), using the Drude $\left[F_{R, D}^{\prime}(a, T)\right]$ and plasma $\left[F_{R, p}^{\prime}(a, T)\right]$ model approaches. The computational results are shown in Fig. 5 by the upper and lower lines, respectively. We emphasize that for two $\mathrm{Ni}$ test bodies $F_{R, D}^{\prime}>F_{R, p}^{\prime}$ at all separations. This is quite the reverse to the case of two Au test bodies and leads to important consequences discussed in Sec. VII. To obtain a striking understanding of the difference between the predictions of the two approaches, in Fig. 6(a) we also plot the difference

$$
F_{\text {diff }}^{\prime}(a, T) \equiv F_{R, D}^{\prime}(a, T)-F_{R, p}^{\prime}(a, T)
$$

at $T=300 \mathrm{~K}$ as a function of separation. In the same figure the dashed line reproduces from Fig. 3 the total experimental error in measurements of force gradients. As can be seen in Fig. 6(a), $F_{\text {diff }}^{\prime}$ is well above the total experimental error determined at a $67 \%$ confidence level up to almost $450 \mathrm{~nm}$. However, with increasing separation distance, the magnitudes 


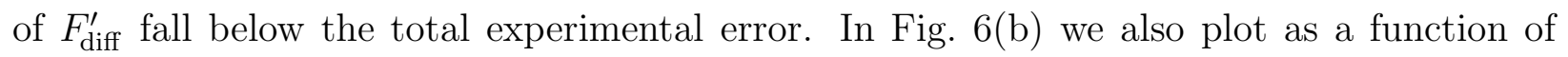
separation the relative difference (in percent) between the predictions of two theoretical approaches $F_{\text {diff }}^{\prime} / F_{R, p}^{\prime}$. It can be seen that the relative difference for $\mathrm{Ni}$ test bodies increases with increasing separation and achieves $10 \%$ at separations above $500 \mathrm{~nm}$ (see Sec. VII where the cases of $\mathrm{Ni}$ and $\mathrm{Au}$ test bodies are compared).

\section{CALCULATION OF MAGNETIC INTERACTION IN THE EXPERIMENTAL SETUP}

Before the measurement data could be compared with the above computational results for the gradient of the Casimir force using different theoretical approaches, due attention should be focused on magnetic interactions. Note that in our experiment both interacting surfaces are ferromagnetic films and consist of many domains. Because of this, it is necessary to calculate the maximum possible contribution of magnetic forces into the measurement results. First, we calculate the energy of magnetic interaction per unit area of two plane parallel films. For this purpose the domain structure of the films of sizes $L_{x}^{(1)} \times L_{y}^{(1)}$ and $L_{x}^{(2)} \times L_{y}^{(2)}$ is periodically continued for infinite planes. Then, using the PFA, we calculate the upper bound for the gradient of the magnetic force acting between a sphere and a plate coated with magnetic films. Note that PFA in the form of Eq. (12) is applicable not only to Casimir forces, but, for instance, to the electric forces which decrease with separation less rapidly. ${ }^{\underline{9}}$ It was shown $\underline{\underline{ }}$ that a more general formulation of the PFA (the so-called Derjaguin method $\left.{ }^{80}\right)$ is applicable even to volumetric forces which do not decrease with separation. In our case of magnetic forces we have checked that the PFA in both formulations leads to coincident results.

The magnetic field created by a magnetic body $V_{1}$ at the point $\left(x_{2}, y_{2}, z_{2}\right)$ is given by the $\operatorname{expression}^{15}$

$$
\boldsymbol{H}\left(x_{2}, y_{2}, z_{2}\right)=\int_{V_{1}} d x_{1} d y_{1} d z_{1} \frac{3\left(\boldsymbol{n}_{r} \cdot \boldsymbol{M}_{1}\right) \boldsymbol{n}_{r}-M_{1}}{|\boldsymbol{r}|^{3}} .
$$

Here, the integration is extended over the coordinates $\left(x_{1}, y_{1}, z_{1}\right)$ of the body $V_{1}$, the unit vector $\boldsymbol{n}_{r}$ is given by

$$
\boldsymbol{n}_{r}=\frac{\boldsymbol{r}}{|\boldsymbol{r}|}
$$


the vector $\boldsymbol{r}$ is directed from the point $\left(x_{1}, y_{1}, z_{1}\right)$ to the point $\left(x_{2}, y_{2}, z_{2}\right)$ and

$$
|\boldsymbol{r}|=\left[\left(x_{2}-x_{1}\right)^{2}+\left(y_{2}-y_{1}\right)^{2}+\left(z_{2}-z_{1}\right)^{2}\right]^{1 / 2} \text {. }
$$

The magnetization distribution $\boldsymbol{M}_{1} \equiv \boldsymbol{M}_{1}\left(x_{1}, y_{1}, z_{1}\right)$ of the body $V_{1}$ depends on a point. The energy of the magnetic interaction between the bodies $V_{1}$ and $V_{2}$ is given by $\underline{15}$

$$
E_{m}=-\int_{V_{2}} d x_{2} d y_{2} d z_{2} \boldsymbol{M}_{2}\left(x_{2}, y_{2}, z_{2}\right) \cdot \boldsymbol{H}\left(x_{2}, y_{2}, z_{2}\right)
$$

where $\boldsymbol{M}_{2} \equiv \boldsymbol{M}_{2}\left(x_{2}, y_{2}, z_{2}\right)$ is the magnetic distribution of the second body.

The orientation of magnetization of separate domains in the magnetic films depends on the film thicknesses. Thus, one can obtain in-plane magnetization only in very thin films. With increasing film thickness up to $150 \mathrm{~nm}$ and more, the easy direction is out-of-plane perpendicular to it $\underline{\underline{81}}$ Although in our experiment the film thicknesses satisfy this condition, below we calculate the upper bounds of the magnetic interaction for both out-of-plane and in-plane magnetizations and show that in both cases the gradient of magnetic force is negligibly small. Note that any other alignment of domains (which cannot occur in thin films but might be possible in thick magnetic bodies) can be presented as a superposition of these two. The results obtained below concerning the smallness of the magnetic interaction are valid for films consisting of many domains. The numerical estimations use the domain sizes, as in our experiment. We start from the most realistic case of an out-of-plane magnetization.

\section{A. Out-of-plane magnetization}

For magnetization perpendicular to the plane of first and second films one has

$$
\boldsymbol{M}_{1,2}=\left(0,0, M_{z}^{(1,2)}\right), \quad M_{z}^{(1,2)} \equiv M_{z}^{(1,2)}\left(x_{1,2}, y_{1,2}\right),
$$

i.e., magnetizations do not vary with film surface distances. We first assume that the magnetizations $M_{z}^{(1,2)}$ take the values $M_{s}$ and $-M_{s}$ with equal probability (it is known that 81

$\left.M_{s}=435 \mathrm{emu} / \mathrm{cm}^{3}\right)$. Here it is assumed that the films do not possess a spontaneous magnetization as predicted by experimental conditions (the case of films possessing a spontaneous magnetization is considered next). The magnetic interaction between the two parallel films of finite area consisting of randomly distributed domains can be calculated using the formalism developed earlier ${ }^{84}$ to take into account the impact of surface roughness to the Casimir 
force. For this purpose we perform the periodic continuation of the functions $M_{z}^{(1,2)}\left(x_{1,2}, y_{1,2}\right)$ as odd functions with periods $2 L_{x}^{(1,2)}$ and $2 L_{y}^{(1,2)}$ over the whole planes $\left(x_{1}, y_{1}\right)$ and $\left(x_{2}, y_{2}\right)$, respectively. The obtained periodic functions can be expanded in the Fourier series

$$
M_{z}^{(1,2)}\left(x_{1,2}, y_{1,2}\right)=\sum_{k, n=1}^{\infty} M_{k n}^{(1,2)} \sin \frac{k \pi x_{1,2}}{L_{x}^{(1,2)}} \sin \frac{n \pi y_{1,2}}{L_{y}^{(1,2)}}
$$

where $M_{k n}^{(1,2)}$ are the Fourier coefficients.

Now one can use the standard formalism of magnetic force microscopy $\frac{85,86}{6}$ to calculate the magnetic energy (22) between the two parallel films spaced at a separation $a$. We emphasize, however, that when scanning a sharp tip above the boundary of two neighboring domains in magnetic force microscopy, they are usually modeled by a periodic structure $.85,87$ In this case the quantities $L_{x, y}^{(1,2)}$ in Eq. (24) are replaced with the characteristic sizes of the magnetic domains $D_{x, y}^{(1,2)}$. For randomly distributed domains, as in our case, the dominant contribution to the right-hand side of Eq. (24) is given by the item numbered with rather large indices $k \approx L_{x}^{(1,2)} / D_{x}^{(1,2)}$ and $n \approx L_{y}^{(1,2)} / D_{y}^{(1,2)}$. Thus, taking into account that the size of magnetic domains is approximately equal to the film thickness, we obtain for the domains on the first film $D_{x}^{(1)} \approx D_{y}^{(1)} \approx d_{1}=250 \mathrm{~nm}$. In a similar way $D_{x}^{(2)} \approx D_{y}^{(2)} \approx d_{2}=210 \mathrm{~nm}$. Then, using the sizes of the first film $L_{x}^{(1)}=0.9 \mathrm{~cm}$ and $L_{y}^{(1)}=1.1 \mathrm{~cm}$, we arrive at $k \approx 3.6 \times 10^{4}$ and $n \approx 4.4 \times 10^{4}$. After calculations using Eqs. (19) $-(\underline{24})$, for the magnetic energy per unit area of the first film one arrives at (see Appendix A for details)

$$
\begin{aligned}
& \frac{E_{m}(a)}{L_{x}^{(1)} L_{y}^{(1)}}=\frac{1}{L_{x}^{(1)} L_{y}^{(1)}} \sum_{k, n=1}^{\infty} \sum_{k^{\prime}, n^{\prime}=1}^{\infty} M_{k n}^{(1)} M_{k^{\prime} n^{\prime}}^{(2)} X_{k k^{\prime}} Y_{n n^{\prime}} \\
& \quad \times \int_{a}^{a+d_{2}} d z_{2}\left[\left(z_{2}+d_{1}\right) \Phi_{k n}\left(z_{2}+d_{1}\right)-z_{2} \Phi_{k n}\left(z_{2}\right)\right] .
\end{aligned}
$$

Here, the functions $X_{k k^{\prime}}$ and $Y_{n n^{\prime}}$ are defined as

$$
\begin{aligned}
& X_{k k^{\prime}}=\int_{0}^{L_{x}^{(1)}} d x \sin \frac{\pi k x}{L_{x}^{(1)}} \sin \frac{\pi k^{\prime} x}{L_{x}^{(2)}}, \\
& Y_{n n^{\prime}}=\int_{0}^{L_{y}^{(1)}} d y \sin \frac{\pi n y}{L_{y}^{(1)}} \sin \frac{\pi n^{\prime} y}{L_{y}^{(2)}}
\end{aligned}
$$

and the function $\Phi_{k n}(z)$ is defined by

$$
\Phi_{k n}(z)=\frac{2 \pi}{z} e^{-\gamma_{k n} z}, \quad \gamma_{k n}=\pi\left[\left(\frac{k}{L_{x}^{(1)}}\right)^{2}+\left(\frac{n}{L_{y}^{(1)}}\right)^{2}\right]^{1 / 2}
$$


Using the PFA in Eq. (12), one can obtain the gradient of the magnetic force acting between a sphere and a plate. For this purpose we replace $\mathcal{F}_{p p}$ in Eq. (12) with $E_{m} /\left(L_{x}^{(1)} L_{y}^{(1)}\right)$ defined in Eq. (25) and perform differentiation with respect to $a$. The result is

$$
\begin{aligned}
& F_{m}^{\prime}(a)=\frac{4 \pi^{2} R}{L_{x}^{(1)} L_{y}^{(1)}} \sum_{k, n=1}^{\infty} \sum_{k^{\prime}, n^{\prime}=1}^{\infty} M_{k n}^{(1)} M_{k^{\prime} n^{\prime}}^{(2)} e^{-\gamma_{k n} a} \\
& \quad \times\left(1-e^{-\gamma_{k n} d_{1}}\right)\left(1-e^{-\gamma_{k n} d_{2}}\right) X_{k k^{\prime}} Y_{n n^{\prime}} .
\end{aligned}
$$

Note that the factors $X_{k k^{\prime}}$ and $Y_{n n^{\prime}}$ do not average to zero due to the finitness of $L_{x}^{(1)}, L_{y}^{(1)}$ and $L_{x}^{(2)} \approx L_{y}^{(2)} \sim 2 R$, i.e., due to the boundary effects.

Now we estimate the gradient of the magnetic force (28) by considering the dominant contribution to Eq. (28). As discussed above, the dominant contribution is given by $k \approx$ $L_{x}^{(1)} / D_{x}^{(1)}$ and $k^{\prime} \approx L_{x}^{(2)} / D_{x}^{(2)}$ and hence by

$$
\begin{aligned}
X_{k k^{\prime}} & =\int_{0}^{L_{x}^{(1)}} d x \sin \frac{\pi x}{D_{x}^{(1)}} \sin \frac{\pi x}{D_{x}^{(2)}} \\
= & \frac{D_{x}^{(1)} D_{x}^{(2)}}{2 \pi}\left\{\frac{1}{D_{x}^{(2)}-D_{x}^{(1)}}\left[\sin \frac{\pi L_{x}^{(1)}}{D_{x}^{(1)}} \cos \frac{\pi L_{x}^{(1)}}{D_{x}^{(2)}}-\cos \frac{\pi L_{x}^{(1)}}{D_{x}^{(1)}} \sin \frac{\pi L_{x}^{(1)}}{D_{x}^{(2)}}\right]\right. \\
& \left.-\frac{1}{D_{x}^{(1)}+D_{x}^{(2)}}\left[\sin \frac{\pi L_{x}^{(1)}}{D_{x}^{(1)}} \cos \frac{\pi L_{x}^{(1)}}{D_{x}^{(2)}}+\cos \frac{\pi L_{x}^{(1)}}{D_{x}^{(1)}} \sin \frac{\pi L_{x}^{(1)}}{D_{x}^{(2)}}\right]\right\} .
\end{aligned}
$$

Taking into account, that $L_{x}^{(1)} / D_{x}^{(1)}$ is an integer number, one obtains

$$
\begin{gathered}
\left|X_{k k^{\prime}}\right| \leq \frac{D_{x}^{(1)} D_{x}^{(2)}}{2 \pi}\left(\frac{1}{D_{x}^{(1)}-D_{x}^{(2)}}-\frac{1}{D_{x}^{(1)}+D_{x}^{(2)}}\right) \\
=\frac{D_{x}^{(1)}}{\pi\left[\left(\frac{D_{x}^{(1)}}{D_{x}^{(2)}}\right)^{2}-1\right]} \approx \frac{D_{x}^{(1)}}{0.4 \pi} \lesssim D_{x}^{(1)} .
\end{gathered}
$$

In a similar way for $n \approx L_{y}^{(1)} / D_{y}^{(1)}, n^{\prime} \approx L_{y}^{(2)} / D_{y}^{(2)}$ we get

$$
\left|Y_{n n^{\prime}}\right| \lesssim D_{y}^{(1)}
$$

Using Eqs. (30) and (31) we calculate the dominant contribution to the gradient of magnetic force (28) at different separations. Thus, at $a=223,250$ and $300 \mathrm{~nm}$ its magnitude is equal to $1.4 \times 10^{-3}, 8.6 \times 10^{-4}$ and $3.5 \times 10^{-4} \mu \mathrm{N} / \mathrm{m}$, respectively. The gradient of the magnetic force further decreases in magnitude with increasing separation. Numerical computations using Eq. (28) show that at all separations the total magnitude of the gradient 
of the magnetic forces due to randomly distributed domains $\left|F_{m}^{\prime}(a)\right|<10^{-2} \mu \mathrm{N} / \mathrm{m}$, i.e., much smaller than the total error in the dynamic measurements of the Casimir interaction (see Sec. III).

The above calculations were performed under an assumption that there is no spontaneous magnetization in our Ni films. Now we include the case that there is some excess in the magnetization of domains in one direction. This can be described by adding a nonzero term $M_{00}^{(1,2)}$ on the right-hand side of Eq. (24) for $M_{z}^{(1,2)}$. The magnetic energy per unit area of two parallel discs of $L_{x}^{(1)} / 2$ radii arising due to such term is obtained as (see Appendix A for details)

$$
E_{s m}(a)=4 \pi M_{00}^{(1)} M_{00}^{(2)} \int_{a}^{a+d_{2}} d z_{2}\left[\frac{z_{2}}{\sqrt{\left(L_{x}^{(1)}\right)^{2}+4 z_{2}^{2}}}-\frac{z_{2}+d_{1}}{\sqrt{\left(L_{x}^{(1)}\right)^{2}+4\left(z_{2}+d_{1}\right)^{2}}}\right] .
$$

Then, the gradient of the magnetic force due to the spontaneous magnetization is found by using the PFA in Eq. (12) where we replace $\mathcal{F}_{p p}$ with $E_{s m}$ defined in Eq. (32). The result is

$$
\begin{gathered}
F_{s m}^{\prime}(a)=8 \pi^{2} R M_{00}^{(1)} M_{00}^{(2)}\left[\frac{a+d_{2}}{\sqrt{\left(L_{x}^{(1)}\right)^{2}+4\left(a+d_{2}\right)^{2}}}-\frac{a}{\sqrt{\left(L_{x}^{(1)}\right)^{2}+4 a^{2}}}\right. \\
\left.-\frac{a+d_{1}+d_{2}}{\sqrt{\left(L_{x}^{(1)}\right)^{2}+4\left(a+d_{1}+d_{2}\right)^{2}}}+\frac{a+d_{1}}{\sqrt{\left(L_{x}^{(1)}\right)^{2}+4\left(a+d_{1}\right)^{2}}}\right] .
\end{gathered}
$$

We calculate the quantity (33) in an extreme case when the magnetic moments of all domains are directed in one direction. In this case $\left|M_{00}^{(1)}\right|=\left|M_{00}^{(2)}\right|=M_{s}$. Then calculations using Eq. (33) result in $\left|F_{s m}^{\prime}\right| \approx 2.6 \times 10^{-5} \mu \mathrm{N} / \mathrm{m}$ at $a=223 \mathrm{~nm}$ and even smaller values at larger separations. Such small magnitudes for the gradient of the magnetic force due to spontaneous magnetization are explained by the fact that in the considered separation region this force depends on separation only slightly. Thus, for out-of-plane magnetization of Ni films one can neglect any influence of the magnetic interaction when measuring the gradient of the Casimir force.

\section{B. In-plane magnetization}

Now we consider the magnetic interaction between Ni-coated surfaces of a plate and a sphere under the assumption that separate domains are characterized by the in-plane magnetization (as discussed above, this might happen for sufficiently thin magnetic films). 
For the in-plane magnetization, one can choose the coordinate system in such a way that for the first film

$$
\boldsymbol{M}_{1}=\left(M_{x}^{(1)}, 0,0\right), \quad M_{x}^{(1)} \equiv M_{x}^{(1)}\left(x_{1}, y_{1}\right)
$$

We further assume that there is no spontaneous magnetization so that $M_{x}^{(1)}= \pm M_{s}$ with equal probability. The in-plane magnetization of the second film may make an angle $\alpha$ with the $x$ axis. Because of this

$$
\boldsymbol{M}_{2}=\left(M_{x}^{(2)}, M_{y}^{(2)}, 0\right)
$$

where both components depend on the position and take random values

$$
M_{x}^{(2)}= \pm M_{s} \cos \alpha, \quad M_{y}^{(2)}= \pm M_{s} \sin \alpha
$$

Similar to Sec. VA, we extrapolate the quantities $M_{x}^{(1)}\left(x_{1}, y_{1}\right)$ and $M_{x, y}^{(2)}\left(x_{2}, y_{2}\right)$ to the entire planes $\left(x_{1}, y_{1}\right)$ and $\left(x_{2}, y_{2}\right)$ as odd functions with the periods $2 L_{x}^{(1,2)}$ and $2 L_{y}^{(1,2)}$, respectively. The obtained periodic functions can be expanded in the Fourier series

$$
\begin{aligned}
& M_{x}^{(1)}\left(x_{1}, y_{1}\right)=\sum_{k, n=1}^{\infty} \tilde{M}_{k n}^{(1)} \sin \frac{k \pi x_{1}}{L_{x}^{(1)}} \sin \frac{n \pi y_{1}}{L_{y}^{(1)}} \\
& M_{x, y}^{(2)}\left(x_{2}, y_{2}\right)=\sum_{k, n=1}^{\infty} \tilde{M}_{x, y ; k n}^{(2)} \sin \frac{k \pi x_{2}}{L_{x}^{(2)}} \sin \frac{n \pi y_{2}}{L_{y}^{(2)}} .
\end{aligned}
$$

After caclulations using Eqs. (19)-(22) and (34)-(37) one obtains an expression for the magnetic energy per unit area of two parallel films (see Appendix B for details)

$$
\begin{aligned}
& \frac{E_{m}(a)}{L_{x}^{(1)} L_{y}^{(1)}}=\frac{2 \pi}{L_{x}^{(1)} L_{y}^{(1)}} \sum_{k, n=1}^{\infty} \sum_{k^{\prime}, n^{\prime}=1}^{\infty} \tilde{M}_{k n}^{(1)}\left\{\tilde{M}_{x ; k^{\prime} n^{\prime}}^{(2)} X_{k k^{\prime}} Y_{n n^{\prime}}\right. \\
& \quad \times \int_{a}^{a+d_{2}} d z_{2}\left[-\frac{k^{2} \pi^{2}}{\left(L_{x}^{(1)}\right)^{2} \gamma_{k n}^{2}} e^{-\gamma_{k n} z_{2}}\left(1-e^{-\gamma_{k n} d_{1}}\right)\right. \\
& \left.\quad-\operatorname{Ei}\left(-\gamma_{k n} z_{2}\right)+\operatorname{Ei}\left[-\gamma_{k n}\left(z_{2}+d_{1}\right)\right]\right] \\
& \left.+\tilde{M}_{y ; k^{\prime} n^{\prime}}^{(2)} \tilde{X}_{k k^{\prime}} \tilde{Y}_{n n^{\prime}} \frac{k n \pi^{2}}{L_{x}^{(1)} L_{y}^{(1)} \gamma_{k n}^{2}}\left(1-e^{-\gamma_{k n} d_{1}}\right) \int_{a}^{a+d_{2}} d z_{2} e^{-\gamma_{k n} z_{2}}\right\}
\end{aligned}
$$

Here, the quantities $X_{k k^{\prime}}$ and $Y_{n n^{\prime}}$ are defined in Eq. (26),$\gamma_{k n}$ is defined in Eq. (27), $\operatorname{Ei}(t)$ 
is the exponential integral and

$$
\begin{aligned}
& \tilde{X}_{k k^{\prime}}=\int_{0}^{L_{x}^{(1)}} d x \sin \frac{\pi k x}{L_{x}^{(1)}} \cos \frac{\pi k^{\prime} x}{L_{x}^{(2)}} \\
& \tilde{Y}_{n n^{\prime}}=\int_{0}^{L_{y}^{(1)}} d y \sin \frac{\pi n y}{L_{y}^{(1)}} \cos \frac{\pi n^{\prime} y}{L_{y}^{(2)}} .
\end{aligned}
$$

The gradient of the magnetic force between a sphere and a plate is obtained from the PFA in Eq. (12) by replacing $\mathcal{F}_{p p}$ with the magnetic energy per unit area defined in Eq. (38). This leads us to the following result:

$$
\begin{aligned}
& F_{m}^{\prime}(a)=\frac{4 \pi^{2} R}{L_{x}^{(1)} L_{y}^{(1)}} \sum_{k, n=1}^{\infty} \sum_{k^{\prime}, n^{\prime}=1}^{\infty} \tilde{M}_{k n}^{(1)}\left\{\tilde{M}_{x ; k^{\prime} n^{\prime}}^{(2)} X_{k k^{\prime}} Y_{n n^{\prime}}\right. \\
& \quad\left[\frac{k^{2} \pi^{2}}{\left(L_{x}^{(1)}\right)^{2} \gamma_{k n}^{2}} e^{-\gamma_{k n} a}\left(1-e^{-\gamma_{k n} d_{1}}\right)\left(1-e^{-\gamma_{k n} d_{2}}\right)\right. \\
& \left.\quad+\operatorname{Ei}\left(-\gamma_{k n} a\right)-\operatorname{Ei}\left[-\gamma_{k n}\left(a+d_{2}\right)\right]-\operatorname{Ei}\left[-\gamma_{k n}\left(a+d_{1}\right)\right]+\operatorname{Ei}\left[-\gamma_{k n}\left(a+d_{1}+d_{2}\right)\right]\right] \\
& \left.\quad-\tilde{M}_{y ; k^{\prime} n^{\prime}}^{(2)} \tilde{X}_{k k^{\prime}} \tilde{Y}_{n n^{\prime}} \frac{k n \pi^{2}}{L_{x}^{(1)} L_{y}^{(1)} \gamma_{k n}^{2}} e^{-\gamma_{k n} a}\left(1-e^{-\gamma_{k n} d_{1}}\right)\left(1-e^{-\gamma_{k n} d_{2}}\right)\right\}
\end{aligned}
$$

Similar to the case of out-of-plane magnetization, the quantity (40) is different from zero only due to the boundary effects. The dominant contribution to Eq. (40) can be estimated using Eqs. (30) and (31) and the same inequalities for $\tilde{X}_{k k^{\prime}}$ and $\tilde{Y}_{n n^{\prime}}$ defined in Eq. (39). As a result, we obtain that at each separation the largest magnitude of $F_{m}^{\prime}$ is achieved at $\alpha=0$, i.e., for the parallel in-plane magnetizations. For example, when separation increases from 223 to $400 \mathrm{~nm}$ the magnitude of $F_{m}^{\prime}$ decreases from $1.1 \times 10^{-3}$ to $5.3 \times 10^{-4} \mu \mathrm{N} / \mathrm{m}$. Thus, in the case of in-plane magnetization the role of magnetic interaction in dynamic measurements of the Casimir force is even smaller than for out-of-plane one.

Now we consider the case that there is a spontaneous magnetization in our Ni films. This can be described by adding nonzero constant terms $M_{00}^{(1)}$ and $M_{x, y ; 00}^{(2)}$ on the right-hand side of Eq. (37). In the same way, as in Sec. VA, for the energy of magnetic interaction per unit 
area of two parallel films we obtain (see Appendix B for details)

$$
\begin{gathered}
E_{s m}(a)=4 M_{00}^{(1)} M_{x, 00}^{(2)} \int_{a}^{a+d_{2}} d z_{2}\left[\arctan \frac{2 z_{2}}{\sqrt{\left(L_{x}^{(1)}\right)^{2}+\left(L_{y}^{(1)}\right)^{2}+4 z_{2}^{2}}}\right. \\
\left.-\arctan \frac{2\left(z_{2}+d_{1}\right)}{\sqrt{\left(L_{x}^{(1)}\right)^{2}+\left(L_{y}^{(1)}\right)^{2}+4\left(z_{2}+d_{1}\right)^{2}}}\right] .
\end{gathered}
$$

Using the PFA in Eq. (12), the gradient of the magnetic force due to spontaneous magnetization takes the form

$$
\begin{aligned}
& F_{s m}^{\prime}(a)=8 \pi R M_{00}^{(1)} M_{x, 00}^{(2)}\left[\arctan \frac{2\left(a+d_{2}\right)}{\sqrt{\left(L_{x}^{(1)}\right)^{2}+\left(L_{y}^{(1)}\right)^{2}+4\left(a+d_{2}\right)^{2}}}\right. \\
& -\arctan \frac{2 a}{\sqrt{\left(L_{x}^{(1)}\right)^{2}+\left(L_{y}^{(1)}\right)^{2}+4 a^{2}}}+\arctan \frac{2\left(a+d_{1}\right)}{\sqrt{\left(L_{x}^{(1)}\right)^{2}+\left(L_{y}^{(1)}\right)^{2}+4\left(a+d_{1}\right)^{2}}} \\
& \left.-\arctan \frac{2\left(a+d_{1}+d_{2}\right)}{\sqrt{\left(L_{x}^{(1)}\right)^{2}+\left(L_{y}^{(1)}\right)^{2}+4\left(a+d_{1}+d_{2}\right)^{2}}}\right] .
\end{aligned}
$$

Assuming that all magnetic moments are directed in one direction (the saturation magnetization), we obtain from Eq. (42) that $\left|F_{s m}^{\prime}\right|$ increases from $1.0 \times 10^{-5}$ to $1.7 \times 10^{-5} \mu \mathrm{N} / \mathrm{m}$ when the separation increases from 223 to $550 \mathrm{~nm}$. This is a negligibly small effect in dynamic measurements of the Casimir force.

\section{COMPARISON BETWEEN EXPERIMENT AND THEORY FOR Ni TEST BODIES}

We have now demonstrated that possible magnetic effect due to the domain structure of $\mathrm{Ni}$ films used in our experiment yields scarcely any contribution to the measured force gradients. Because of this the measurement results for the gradients of the Casimir force presented in Sec. III can be reliably compared with the predictions of the Lifshitz theory taking into account nonzero temperature, conductivity properties of $\mathrm{Ni}$, surface roughness and inaccuracy of the PFA, as discussed in Sec. IV.

In Ref. $\underline{49}$ we have used the traditional method of the comparison between experiment and theory when the measurement data are presented as crosses whose horizontal arms are equal to $2 \Delta a$ and the vertical arms are equal to $2 \Delta^{t} F^{\prime}(a)$. Here we use another method of 
comparison $\underline{9}, 10.44,53$ based on consideration of the confidence interval for the random quantity $F_{R}^{\prime}\left(a_{i}\right)-\bar{F}^{\prime}\left(a_{i}\right)$ equal to the difference between theoretical and mean experimental force gradients at the experimental separations $a_{i}$. This method is advantageous because it allows to make the quantitative conclusions not only about the rejection of any theoretical approach, but about the measure of agreement between experiment and theory as well.

To calculate the confidence interval for the difference between theoretical and mean experimental force gradients, one needs to have the total errors of both quantities. The total experimental error $\Delta^{t} F^{\prime}$ is already determined in Sec. III (see Fig. 3). The crucial contribution to the theoretical error is given by the errors in optical data of Ni determined by the number of significant figures in the tables. $\underline{\underline{54}}$ The errors in the optical data lead to the theoretical error $\Delta^{\text {opt }} F_{R}^{\prime}$ equal to approximately $0.5 \%$ of $F_{R}^{\prime}$ (it is shown by the long-dashed line in Fig. 7 as a function of separation). There is, however, one more source of error ${ }^{89}$ when the theoretical value of the force gradient is calculated not over some separation interval but at the experimental separations $a_{i}$. The point is that each experimental separation is determined up to an error $\Delta a$ and this leads to a respective error in the calculated force gradients $\underline{89}$

$$
\Delta^{\mathrm{sep}} F_{R}^{\prime}\left(a_{i}\right) \approx 4 \frac{\Delta a}{a_{i}} F_{R}^{\prime}\left(a_{i}\right) .
$$

In Fig. 7 the theoretical error $\Delta^{\text {sep }} F_{R}^{\prime}$ as a function of separation is shown by the short-dashed line. In the same figure the solid line shows the total theoretical error $\Delta^{t} F_{R}^{\prime}$ determined at a $67 \%$ confidence level which was combined in quadrature from the theoretical errors $\Delta^{\mathrm{opt}} F_{R}^{\prime}$ and $\Delta^{\mathrm{sep}} F_{R}^{\prime}$. The total theoretical error varies from 0.99 to $0.03 \mu \mathrm{N} / \mathrm{m}$ when the separation increases from 223 to $500 \mathrm{~nm}$. As can be seen from the comparison with Fig. 3, at all separations the total experimental error is in exceess of the total theoretical error. As a result, the confidence interval for the quantityv $F_{R}^{\prime}\left(a_{i}\right)-\bar{F}^{\prime}\left(a_{i}\right)$ determined at a $67 \%$ confidence level is given by $\left[-\Xi_{F^{\prime}}^{0.67}(a), \Xi_{F^{\prime}}^{0.67}(a)\right]$, where

$$
\Xi_{F^{\prime}}^{0.67}(a)=\left\{\left[\Delta^{t} F^{\prime}(a)\right]^{2}+\left[\Delta^{t} F_{R}^{\prime}(a)\right]^{2}\right\}^{1 / 2} .
$$

In Fig. 8 (a) we show the quantities $\pm \Xi_{F^{\prime}}^{0.67}(a)$ as functions of separation by the solid lines. In doing so the confidence interval at each fixed $a$ is the vertical segment between $-\Xi_{F^{\prime}}^{0.67}(a)$ and $\Xi_{F^{\prime}}^{0.67}(a)$. It has the meaning that if the theory is consistent with the data then at least $67 \%$ data points within each separation subinterval must belong to this confidence interval. To verify which of the two theoretical approaches used in Sec. IV is consistent with the 
data, in Fig. $8(\mathrm{a}, \mathrm{b})$ we plot the differences $F_{R}^{\prime}\left(a_{i}\right)-\bar{F}^{\prime}\left(a_{i}\right)$ as black and gray dots, where the plasma model and the Drude model approaches, respectively, were used to compute the quantity $F_{R}^{\prime}\left(a_{i}\right)$ (see the lower and upper lines in Fig. [5). As can be seen in Fig. 8(a), not only $67 \%$ but all black dots belong to the confidence intervals within the entire separation region from 223 to $550 \mathrm{~nm}$. This means that the data are consistent with the Lifshitz theory combined with the plasma model approach with a large safety margin. As to the gray dots, most of them are outside the confidence intervals over the separation region from 223 to $420 \mathrm{~nm}$. Thus, the Drude model approach to the Casimir force is excluded by the data within this separation region at a $67 \%$ confidence level.

As is seen in Fig. 8(a), even if the confidence intervals were widened to reach a $95 \%$ confidence level, the differences $F_{R, D}^{\prime}\left(a_{i}\right)-\bar{F}^{\prime}\left(a_{i}\right)$ computed using the Drude model approach would still remain outside those intervals within some range of separations. To make this observation quantitative, we calculate the half-width of the confidence interval $\Xi_{F^{\prime}}^{0.95}(a)$ from the equation

$$
\frac{\Xi_{F^{\prime}}^{0.95}(a)}{\Xi_{F^{\prime}}^{0.67}(a)}=\frac{t_{(1+0.95) / 2}(32)}{t_{(1+0.67) / 2}(32)} \approx 2 .
$$

In Fig. 8(b) we plot the borders of the $95 \%$ confidence intervals $\pm \Xi_{F^{\prime}}^{0.95}(a)$ by the solid lines and reproduce the black and gray dots from Fig. 8(a). As can be seen in Fig. 8(b), the most of gray dots are still outside the widened confidence intervals within the separation interval from 223 to $345 \mathrm{~nm}$. This allows one to conclude that at these separations the Drude model approach is excluded by the data at a higher, 95\%, confidence level.

To give a better understanding of the character of agreement (disagreement) between the nonaveraged data and two theoretical approaches, in Fig. 9 we provide a histogram plotted at $a=251 \mathrm{~nm}$. Here, $f$ is the fraction of 33 data points having $F^{\prime}$ in the bin shown by the respective vertical lines. The data are consistent with the Gaussian distribution with the standard deviation $\sigma_{F^{\prime}}=0.92 \mu \mathrm{N} / \mathrm{m}$ and the mean gradient $\bar{F}^{\prime}=74.17 \mu \mathrm{N} / \mathrm{m}$ shown by the dashed line. The black and gray vertical lines in Fig. 9] show the theoretical predictions of the plasma model, $F_{R, p}^{\prime}=74.19 \mu \mathrm{N} / \mathrm{m}$, and the Drude model, $F_{R, D}^{\prime}=77.46 \mu \mathrm{N} / \mathrm{m}$, approaches, respectively. Note that in Ref. $\underline{49}$ there is a typo in the value of separation $(250 \mathrm{~nm}$ instead of $251 \mathrm{~nm}$ in the inset to Fig. 2). It is seen that the plasma model approach is in a very good agreement with the measurement result as

$$
F_{R, p}^{\prime}-\bar{F}^{\prime}=0.02 \mu \mathrm{N} / \mathrm{m}<\frac{1}{52} \Delta^{t} F^{\prime}
$$


At the same time the theoretical prediction of the Drude model approach is excluded at high confidence as

$$
F_{R, D}^{\prime}-\bar{F}^{\prime}=3.29 \mu \mathrm{N} / \mathrm{m}>3 \Delta^{t} F^{\prime} .
$$

In the end of this section we emphasize that although Figs. 8(a) and (b) allow the exclusion of the Drude model approach in a quantitative way (at $67 \%$ and $95 \%$ confidence levels within respective separation regions), they cannot be considered as a confirmation of the plasma model approach at either $67 \%$ or $95 \%$ confidence level. The situation here is just the opposite: the higher is the confidence level at which the Drude model is excluded [for example, $95 \%$ in Fig. 8(b)], the easier is for the plasma model approach to accomodate all the points for the gradient differences within the widened confidence interval. In fact, to obtain the quantitative description for the measure of agreement between some experimentally consistent theoretical approach and the data one should make the confidence interval as narrow as possible and determine the respective low confidence level at which this approach is excluded by the data. Then one can conclude that the theoretical approach under consideration is confirmed by the data at a complementary to $100 \%$ high confidence.

To illustrate the above, let us consider the confidence interval $\left[-\Xi_{F^{\prime}}^{0.1}(a), \Xi_{F^{\prime}}^{0.1}(a)\right]$ defined at a $10 \%$ confidence. This can be found from the equality

$$
\frac{\Xi_{F^{\prime}}^{0.1}(a)}{\Xi_{F^{\prime}}^{0.67}(a)}=\frac{t_{(1+0.1) / 2}(32)}{t_{(1+0.67) / 2}(32)} \approx 0.13 .
$$

In Fig. 10 the borders of the 10\% confidence intervals are plotted as the two solid lines and the black dots show the same differences $F_{R, p}^{\prime}\left(a_{i}\right)-\bar{F}^{\prime}\left(a_{i}\right)$ as are shown by the black dots in Fig. 8. As can be seen in Fig. 10, in spite of rather narrow 10\% confidence intervals, much more than $10 \%$ of all dots within any separation subinterval belong to them. This means that the plasma model approach is not excluded by the data even at a $10 \%$ confidence level or, equivalently, that this approach is confirmed by the data at more than $90 \%$ confidence level.

As mentioned in Secs. I and IV, it remains unclear why the experimental data are in agreement with theory disregarding really existing relaxation properties of conduction electrons and exclude the theory taking these properties into account. In the next section we compare the above experimental results for two Ni films with respective measurements in configurations containing one or two Au test bodies. We show that the unique feature of two Ni test bodies shown in Fig. $5\left(F_{R, D}^{\prime}>F_{R, p}^{\prime}\right)$ leads to important conclusions with respect to 
the role of possible background effects in measurements of the Casimir force, such as patch potentials. $\underline{90}$

\section{COMPARISON WITH EXPERIMENTS INVOLVING NONMAGNETIC METALS}

Here we compare the experimental results and the measure of their agreement with theory for two Ni test bodies with the results of previous measurements using the same setup. One of them was performed $\underline{40,91}$ with an Au-coated plate and an Au-coated sphere, and the other $^{33}$ with a Ni-coated plate and an Au-coated sphere.

We begin from the experiment $\underline{40}, \underline{1}$ using an Au-coated plate and an Au-coated sphere of $R=41.3 \mu \mathrm{m}$ radius. First, we present the results of this experiment in terms of the differences between the theoretical force gradients computed using either the Drude model or the plasma model approaches and mean measured gradients of the Casimir force. These differences are shown in Fig. 11(a,b) by the gray and black dots, respectively. Note that for $\mathrm{Au}$ computations using the Drude model approach have been made ${ }^{40}, 91$ with the tabulated optical data ${ }^{54}$ extrapolated to zero frequency by the Drude model (15) with the parameters $\omega_{p}=9.0 \mathrm{eV}$ and $\gamma=0.035 \mathrm{eV}$. Recently it was $\operatorname{shown}^{92}$ that $\varepsilon\left(i \xi_{l}\right)$ obtained in this way is in excellent agreement with the dielectric permittivity obtained by means of the weighted Kramers-Kronig relations from the tabulated optical data ${ }^{54}$ with no extrapolation. Furthermore, ellipsometry measurements of the optical properties of Au films were found ${ }^{93}$ in good agreement with the results of Ref. $\underline{54}$. The alternative optical data for Au contained in

the literature, which can significantly deviate from the tabulated data, $\underline{54}$ were shown $\underline{9}, \underline{10}$ to lead to much larger deviations between the predictions of the Drude model approach and measurements of the Casimir force than the data of Ref. $\underline{\underline{54}}$.

The solid lines in Fig. 11 indicate the borders of the confidence intervals determined at (a) 67\% confidence and (b) 95\% confidence level. They are found by using the total experimental and theoretical errors in the experiment of Refs $\underline{40}, \underline{91}$, as discussed in Sec. VI of the present paper. As can be seen in Fig. 11, the plasma model approach is consistent with the data over the entire separation region. As to the Drude model approach, it is excluded by the data at a $67 \%$ confidence level over the separation region from 235 to $420 \mathrm{~nm}$ [see Fig. 11(a)] and at a 95\% confidence level over the separation region from 235 to $330 \mathrm{~nm}$ [see 
Fig. 11(b)].

From the comparison of Figs. 8 and 11 one can observe an important difference between the cases of Ni-Ni and $\mathrm{Au}$-Au test bodies. Note that for Ni-Ni test bodies $F_{R, D}^{\prime}-\bar{F}^{\prime}>0$ (see Fig. (8), i.e.., $F_{R, D}^{\prime}>F_{R, p}^{\prime}$, in contrast to the case of Au-Au test bodies where $F_{R, D}^{\prime}-\bar{F}^{\prime}<0$ (see Fig. 11) and $F_{R, D}^{\prime}<F_{R, p}^{\prime}$. This difference sheds light on the possible role and size of electrostatic patches in measurements of the Casimir force. It was hypothesized $\underline{94}$ that an additional attractive force due to the effect of large patches might bring the experimental data for the two $\mathrm{Au}$ test bodies in agreement with the predictions of the Drude model approach. From Fig. 11 it is seen that the attractive force with a magnitude equal to the difference between two sets of dots would really bring the gray dots in agreement and the black dots in disagreement with the data. It is not logical, however, to assume that the patch effect plays this role for Au but does not play the same role for Ni. From Fig. 8 it follows that any additional attractive force would only increase the disagreement of the Drude model approach with the data leading also to a disagreement of the plasma model approach with the same data. This is in favor of the statement that surface patches lead to only a negligibly small effect in measurements of the Casimir interaction by means of AFM and micromachined oscillator $\underline{\underline{9}} \underline{10,44}$ in qualitative agreement to the model of patches proposed in Ref. $\underline{90}$. This conlusion was recently confirmed $\underline{95}$ by means of Kelvin probe microscopy.

Futher confirmation for a negligibly small role of the effect of electrostatic patches in measurements of the Casimir interaction by means of an AFM comes from the experiment ${ }^{33}$ with a Ni-coated plate and an Au-coated sphere of $R=64.1 \mu$ m radius. In this configuration the predictions of both theoretical approaches to the Casimir force are almost coincident over the experimental separations range. To see this in Fig. 12(a) we show the quantity $F_{\text {diff }}^{\prime}(a) / R$ [see Eq. (18)] by the three solid lines from top to bottom for the experiments on measuring the gradient of the Casimir force between Ni-Ni (this work), Ni-Au (Ref. $\frac{33}{}$ ) and $\mathrm{Au}-\mathrm{Au}$ test bodies,$\underline{40,91}$ respectively (in each case the respective value of the sphere radius is used to make the presented results comparable). To gain a better understanding of distinctions between the two theoretical approaches, in Fig. 12(b) we also show the quantity $F_{\text {diff }}^{\prime}(a) / F_{R, p}^{\prime}(a)$ in percent for the three experiments in the same succession as in Fig. 12(a). As is seen in Fig. 12(a,b), for Ni-Au test bodies (the lines sandwiched between the top and bottom ones) the quantity $F_{\text {diff }}^{\prime} / R$ and $F_{\text {diff }}^{\prime} / F_{R, p}^{\prime}$ cannot be distinguished from zero in the limits of experimental errors. However, for all the three experiments, including that with 
$\mathrm{Ni}-\mathrm{Au}$ test bodies, the measurement results are consistent with theoretical predictions using the plasma model approach. This is seen in Fig. 13)(a,b) where, to make the results of the different experiments comparable, the quantity $F_{R, p}^{\prime} / R$ is shown by the solid dark bands and the crosses represent measurement data with their total experimental errors normalized by the radii (the bands having thicknesses equal to twice the theoretical error are again plotted from top to bottom for experiments with $\mathrm{Au}-\mathrm{Au}, \mathrm{Ni}-\mathrm{Au}$, and $\mathrm{Ni}-\mathrm{Ni}$ test bodies, respectively). Remembering that for $\mathrm{Ni}-\mathrm{Au}$ test bodies two alternative theoretical approaches lead to almost coincident predictions, an introduction of some detectable additional force originating, for instance, from patch potentials would inevitably make both approaches inconsistent with the experimental data.

In Fig. 14 we demonstrate that it is impossible to simultaneously reconcile the Drude model approach with the data of two experiments using $\mathrm{Au}-\mathrm{Au}$ and $\mathrm{Ni}-\mathrm{Ni}$ test bodies at the expense of any unaccounted hypothetical background effect leading to either attractive or repulsive force. In this figure the upper and lower bands show the theoretical results obtained using the Drude model approach for the quantity $F_{R, D}^{\prime} / R$ for $\mathrm{Au}-\mathrm{Au}$ and $\mathrm{Ni}-\mathrm{Ni}$ test bodies, respectively. As can be seen in Fig. 14, there is an evident inconsistency between the data of both experiments and theoretical predictions of the Drude model approach. The important point is that to remedy the problem one would need to introduce some hypothetical attractive

force for the experiment with Au-Au test bodies (the upper band and set of crosses) and a hypothetical repulsive force for the experiment with $\mathrm{Ni}-\mathrm{Ni}$ test bodies (the lower band and set of crosses). Thus, not only an electrostatic attraction due to patch potentials, but any unaccounted hypothetical interaction preserving its sign (i.e., being either attractive or repulsive) is incapable to reconcile the predictions of the Drude model approach with the data. Keeping in mind that in Sec. V we have carefully examined possible contributions of magnetic interactions due to the domain structure of $\mathrm{Ni}$ films and found it negligibly small, any alternative interpretation of our measurement results faces severe difficulties.

\section{CONCLUSIONS AND DISCUSSION}

In the foregoing we have presented complete calibration and measurement data of the experiment on measuring the gradient of the Casimir force between a Ni-coated plate and a Ni-coated sphere by means of dymanic AFM operated in the frequency shift technique. 
This is the pioneering experiment which measured the influence of magnetic properties of the boundary metals on the Casimir interaction predicted theoretically more than 40 years ago. Taking into account that the magnitudes of the force gradients under consideration are about or less than $100 \mu \mathrm{N} / \mathrm{m}$ and the magnetic properties contribute up to $5 \%$ of this quantity, it becomes clear that such experiments call for extreme care to the vacuum system, surface preparation, calibration procedures, background effects, error analysis and comparison between experiment and theory. In this paper we have presented exhaustive information on all the above subjects which has not been already elucidated in the papers devoted to previous experiments using the same setup with $\mathrm{Au}-\mathrm{Au}$ and $\mathrm{Au}-\mathrm{Ni}$ test bodies, $, 33,40,91$ and with $\mathrm{Ni}-\mathrm{Ni}$ test bodies published only in Letter form. $\underline{\underline{49}}$

After a brief description of some details of the setup which were not described in the literature so far, we have presented the results of the electrostatic calibrations which allow precise determination of the calibration constant, closest absolute separation and residual potential difference. All the details of error analysis, including the random, systematic errors and their combination into the total error, were provided. Both individual measured gradients of the Casimir force and their mean values were presented. Computations of the gradients of the Casimir force in the sphere-plane geometry were performed using the Lifshitz theory at nonzero temperature taking into account the recently calculated correction terms to the PFA and the surface roughness. The conductivity properties of $\mathrm{Ni}$ were described in succession using the Drude and the plasma model approaches to the Casimir force presented in the literature and the obtained results were compared between themselves and with the total experimental errors.

We have investigated possible magnetic interaction between the test bodies in our experimental configuration arising due to the domain structure of Ni films. Both cases, out-of-plane and in-plane magnetizations, have been studied extensively (the former has been only briefly considered with respect to measurements of the Casimir force ${ }^{49}$ and the latter was not previously investigated). Although extreme care has been taken in order to avoid spontaneous magnetization of the Ni films used, the case of the fully magnetized films was also considered. It was shown that in all cases the contribution of magnetic interaction to the measured force gradient is by several orders of magnitude smaller than the total experimental error. This allowed a reliable comparison of the measured gradients of the Casimir force with theoretical predictions. 
The comparison of the experimental results with theory was based on a more rigorous method different from that used in Refs. $33,40,49,91$. This method is based on the consideration of the random quantity equal to the difference between theoretical and mean experimental force gradients. Both $67 \%$ and $95 \%$ confidence intervals for this quantity were found. The preference of the comparison method under discussion is that it not only allows one to exclude some theoretical approach as inconsistent with the data at a given confidence level, but also permits to quantitatively determine at what confidence level a theoretical approach is confirmed by the data. On this basis we have concluded that the Drude model approach to the Casimir force is excluded by our measurements with two Ni surfaces at a $95 \%$ confidence level, whereas the plasma model approach is confirmed by the data at higher than $90 \%$ confidence level. In this work we have investigated in detail the striking property of the Casimir interaction between two magnetic test bodies, i.e., that the force gradients calculated using the Drude model approach are significantly larger than the measured mean force gradients. This is just the opposite of the case of two nonmagnetic $(\mathrm{Au})$ test bodies where the theoretical force gradients, calculated using the Drude model approach are significantly smaller than the measured mean force gradients. By comparing the measurement results of the three experiments with $\mathrm{Au}-\mathrm{Au}, \mathrm{Ni}-\mathrm{Au}$, and $\mathrm{Ni}-\mathrm{Ni}$ test bodies taking the above property into account, we have arrived at the conclusion of major importance that no hypothetical unaccounted background force (either attractive or repulsive) could bring the measurement data into agreement with theoretical predictions of the Drude model approach (the attractive force arising due to electrostatic patches is only one example of possible interactions). This means that an exclusion of the Drude model approach by the data assumes a greater significance which awaits for its fundamental explanation. $\underline{\underline{73}}$

To conclude we would like to stress that the experiment on measuring the gradient of the Casimir force between two Ni surfaces has brought confirmation to the prediction of the Lifshitz theory that magnetic properties of boundary surfaces influence the Casimir force. According to our measurement results, the quantitative description of the Casimir interaction between both magnetic and nonmagnetic metals is given by the plasma model approach. At this point it is pertinent to note that in the configuration of a ferromagnetic dielectric interacting with a nonmagnetic metal described by the plasma model the Lifshitz theory predicts the Casimir repulsion through a vacuum gap $\underline{\underline{24}-26}$ This makes possible realization of the Casimir repulsion on microscales in the near future for subsequent applications to the 
problems of lubrication and friction in nanodevices.

\section{Acknowledgments}

The authors are grateful to G. Bimonte for providing the numerical values of a correction beyond the PFA at room temperature from Fig. 2 of Ref $\frac{77}{}$ and to L. P. Teo for providing the numerical values of the same correction at $T=0$ from Fig. 9 of Ref. $\underline{78}$. This work was supported by the DOE Grant No. DEF010204ER46131 (equipment, G.L.K., V.M.M., U.M.).

\section{APPENDIX A}

In this Appendix we derive some mathematical results used in Sec. VA to calculate the magnitude of magnetic interactions in our experimental setup for out-of-plane magnetized Ni films.

From Eq. (19) we can find the z-component of the magnetic field created by the periodically extended first $\mathrm{Ni}$ film at the points of the second $\mathrm{Ni}$ film

$$
H_{z}\left(x_{2}, y_{2}, z_{2}\right)=\int_{-\infty}^{\infty} d x_{1} \int_{-\infty}^{\infty} d y_{1} \int_{-d_{1}}^{0} d z_{1}\left[\frac{3\left(z_{2}-z_{1}\right)^{2}}{|\boldsymbol{r}|^{5}}-\frac{1}{|\boldsymbol{r}|^{3}}\right] M_{z}^{(1)}\left(x_{1}, y_{1}\right),
$$

where the radius-vector $\boldsymbol{r}$ is defined in Eq. (21) and the magnetization is specified in Eq. (23). Calculating the integral with respect to $z_{1}$ in Eq. (A1), we obtain

$$
\begin{gathered}
H_{z}\left(x_{2}, y_{2}, z_{2}\right)=\int_{-\infty}^{\infty} d x_{1} \int_{-\infty}^{\infty} d y_{1}\left\{\frac{z_{2}}{\left[z_{2}^{2}+\left(x_{2}-x_{1}\right)^{2}+\left(y_{2}-y_{1}\right)^{2}\right]^{3 / 2}}\right. \\
\left.-\frac{z_{2}+d_{1}}{\left[\left(z_{2}+d_{1}\right)^{2}+\left(x_{2}-x_{1}\right)^{2}+\left(y_{2}-y_{1}\right)^{2}\right]^{3 / 2}}\right\} M_{z}^{(1)}\left(x_{1}, y_{1}\right) .
\end{gathered}
$$

Now we assume that there is no spontaneous magnetization and substitute the Fourier series (24) in Eq. (A2). After introducing the new variables $u=x_{1}-x_{2}$ and $v=y_{1}-y_{2}$, transforming the sinus functions and equating to zero the integrals of odd functions, Eq. (A2) can be brought to the form

$$
\begin{array}{r}
H_{z}\left(x_{2}, y_{2}, z_{2}\right)=\sum_{k, n=1}^{\infty} M_{k n}^{(1)} \sin \frac{k \pi x_{2}}{L_{x}^{(1)}} \sin \frac{n \pi y_{2}}{L_{y}^{(1)}} \\
\times\left[z_{2} \Phi_{k n}\left(z_{2}\right)-\left(z_{2}+d_{1}\right) \Phi_{k n}\left(z_{2}+d_{1}\right)\right] .
\end{array}
$$


Here we have introduced the notation

$$
\Phi_{k n}(z)=\int_{-\infty}^{\infty} d u \int_{-\infty}^{\infty} d v \frac{\cos \frac{k \pi u}{L_{x}^{(1)}} \cos \frac{n \pi v}{L_{y}^{(1)}}}{\left(u^{2}+v^{2}+z^{2}\right)^{3 / 2}} .
$$

The double integral in Eq. (A4) can be evaluated explicitly. For this purpose we set $\pi k / L_{x}^{(1)}=a_{k}, \pi n / L_{y}^{(1)}=b_{k}$ and calculate the derivative ${ }^{8 \underline{8}}$

$$
\begin{aligned}
\frac{d \Phi_{k n}(z)}{d b_{n}} & =-2 \int_{-\infty}^{\infty} d u \cos \left(a_{k} u\right) \int_{0}^{\infty} d v \frac{v \sin \left(b_{n} v\right)}{\left(u^{2}+z^{2}+v^{2}\right)^{3 / 2}} \\
& =-4 b_{n} \int_{-\infty}^{\infty} d u \cos \left(a_{k} u\right) K_{0}\left(b_{n} \sqrt{z^{2}+u^{2}}\right) \\
& =-2 \pi \frac{b_{n}}{\gamma_{k n}} e^{-\gamma_{k n} z},
\end{aligned}
$$

where $K_{0}(t)=(\pi i / 2) H_{0}^{(1)}(i t)$ is the Bessel function of imaginary argument and $\gamma_{k n}$ is defined in Eq. (27). Then by the integration of Eq. (A5) with respect to $b_{n}$ one finds

$$
\Phi_{k n}(z)=\frac{2 \pi}{z} e^{-\gamma_{k n} z}+G\left(a_{k}, z\right),
$$

where $G\left(a_{k}, z\right)$ is the integration constant. The value of this constant can be found by considering the quantity (A4) with $n=b_{n}=0$

$$
\begin{aligned}
\Phi_{k 0}(z) & =4 \int_{0}^{\infty} d u \cos \left(a_{k} u\right) \int_{0}^{\infty} \frac{d v}{\left(u^{2}+z^{2}+v^{2}\right)^{3 / 2}} \\
& =4 \int_{0}^{\infty} d u \frac{\cos \left(a_{k} u\right)}{z^{2}+u^{2}}=\frac{2 \pi}{z} e^{-z a_{k}} .
\end{aligned}
$$

Comparing this with Eq. (A6), we can conclude that $G\left(a_{k}, z\right)=0$. Thus, from (A6) one arrives at the final expression (27) for the function $\Phi_{k n}(z)$.

The energy of magnetic interaction between parallel plates can be now obtained from Eq. (22)

$$
E_{m}(a)=-\int_{0}^{L_{x}^{(1)}} d x_{2} \int_{0}^{L_{y}^{(1)}} d y_{2} \int_{a}^{a+d_{2}} d z_{2} M_{z}^{(2)}\left(x_{2}, y_{2}\right) H_{z}\left(x_{2}, y_{2}, z_{2}\right) .
$$

Substituting here with Eq. (24) for the magnetization of the second film, Eq. (A3) for the magnetic field and using notations (26)), one arrives at the expression in Eq. (25)).

If the spontaneous magnetizaion is present, Eq. (A2) for the respective magnetic field created by the first film, should be rewritten in the form

$$
\begin{gathered}
H_{z}\left(x_{2}, y_{2}, z_{2}\right)=M_{00}^{(1)} \int_{-L_{x}^{(1)} / 2}^{L_{x}^{(1)} / 2} d x_{1} \int_{-L_{y}^{(1)} / 2}^{L_{y}^{(1)} / 2} d y_{1} \\
\left.-\frac{z_{2}+d_{1}}{\left[\left(z_{2}+d_{1}\right)^{2}+\left(x_{2}-x_{1}\right)^{2}+\left(y_{2}-y_{1}\right)^{2}\right]^{3 / 2}}\right\} .
\end{gathered}
$$


Now we take into account that the second film is situated above the center of a large plate, i.e., $x_{2}<<L_{x}^{(1)}$ and $y_{2}<<L_{y}^{(1)}$. Thus, with sufficient precision one can put $x_{2} \approx y_{2} \approx 0$. Replacing the first film with a disc of $L_{x}^{(1)} / 2=0.5 \mathrm{~cm}$ radius, we obtain the following estimate

$$
H_{z}\left(z_{2}\right) \approx 4 \pi M_{00}^{(1)}\left[\frac{z_{2}+d_{1}}{\sqrt{\left(L_{x}^{(1)}\right)^{2}+4\left(z_{2}+d_{1}\right)^{2}}}-\frac{z_{2}}{\sqrt{\left(L_{x}^{(1)}\right)^{2}+4 z_{2}^{2}}}\right] .
$$

Then, calculating the magnetic energy arising per unit film area due to the spontaneous magnetization

$$
E_{s m}(a)=-M_{00}^{(2)} \int_{a}^{a+d_{2}} d z_{2} H_{z}\left(z_{2}\right)
$$

we arrive at Eq. (32).

\section{APPENDIX B}

Here we derive the mathematical expressions used in Sec. VB to calculate the gradient of magnetic force for the case of in-plane magnetization of $\mathrm{Ni}$ films.

We begin from calculation of the $x$ - and $y$-components of magnetic field created by the periodically continued first $\mathrm{Ni}$ film at the points of parallel to it second Ni film. From Eq. (19) for the in-plane magnetization one obtains

$$
\begin{aligned}
& H_{x}\left(x_{2}, y_{2}, z_{2}\right)=\int_{-\infty}^{\infty} d x_{1} \int_{-\infty}^{\infty} d y_{1} \int_{-d_{1}}^{0} d z_{1}\left[\frac{3\left(x_{2}-x_{1}\right)^{2}}{|\boldsymbol{r}|^{5}}-\frac{1}{|\boldsymbol{r}|^{3}}\right] M_{x}^{(1)}\left(x_{1}, y_{1}\right), \\
& H_{y}\left(x_{2}, y_{2}, z_{2}\right)=\int_{-\infty}^{\infty} d x_{1} \int_{-\infty}^{\infty} d y_{1} \int_{-d_{1}}^{0} d z_{1} \frac{3\left(x_{2}-x_{1}\right)\left(y_{2}-y_{1}\right)}{|\boldsymbol{r}|^{5}} M_{x}^{(1)}\left(x_{1}, y_{1}\right),
\end{aligned}
$$

where the magnetization is presented in Eq. (37). Let us calculate the component $H_{y}$ first. For this purpose we use the identity

$$
\frac{3\left(x_{2}-x_{1}\right)}{|\boldsymbol{r}|^{5}}=-\frac{\partial}{\partial x_{2}} \frac{1}{\left[\left(x_{2}-x_{1}\right)^{2}+\left(y_{2}-y_{1}\right)^{2}+\left(z_{2}-z_{1}\right)^{2}\right]^{3 / 2}} .
$$

Substituting Eqs. (Bי Appendix A, we find

$$
\begin{aligned}
& H_{y}\left(x_{2}, y_{2}, z_{2}\right)=-\sum_{k, n=1}^{\infty} \tilde{M}_{k n}^{(1)} \frac{\partial}{\partial x_{2}} \int_{-\infty}^{\infty} d u \int_{-\infty}^{\infty} d v \int_{-d_{1}}^{0} d z_{1} \\
& \times \frac{v \sin \left[a_{k}\left(u+x_{2}\right)\right] \sin \left[b_{n}\left(v+y_{2}\right)\right]}{\left[u^{2}+v^{2}+\left(z_{2}-z_{1}\right)^{2}\right]^{3 / 2}}
\end{aligned}
$$


where $a_{k}$ and $b_{n}$ are defined in Appendix A below Eq. (A4). Now we transform the sinus functions, set equal to zero the integrals of odd functions, and calculate the derivative with respect to $x_{2}$. The result is

$$
\begin{aligned}
& H_{y}\left(x_{2}, y_{2}, z_{2}\right)=-\sum_{k, n=1}^{\infty} \tilde{M}_{k n}^{(1)} a_{k} \cos \left(a_{k} x_{2}\right) \cos \left(b_{n} y_{2}\right) \\
& \quad \times \int_{-d_{1}}^{0} d z_{1} \int_{-\infty}^{\infty} d u \int_{-\infty}^{\infty} d v \frac{v \cos \left(a_{k} u\right) \sin \left(b_{n} v\right)}{\left[u^{2}+v^{2}+\left(z_{2}-z_{1}\right)^{2}\right]^{3 / 2}}
\end{aligned}
$$

Using the differentiation with respect to $b_{n}$ and the notation (A4), Eq. (B4) can be identically presented in the form

$$
\begin{aligned}
& H_{y}\left(x_{2}, y_{2}, z_{2}\right)=\sum_{k, n=1}^{\infty} \tilde{M}_{k n}^{(1)} a_{k} \cos \left(a_{k} x_{2}\right) \cos \left(b_{n} y_{2}\right) \\
& \quad \times \int_{-d_{1}}^{0} d z_{1} \frac{\partial}{\partial b_{n}} \Phi_{k n}\left(z_{2}-z_{1}\right) .
\end{aligned}
$$

Substituting here Eq. (A5) one obtains after some transformations

$$
\begin{aligned}
& H_{y}\left(x_{2}, y_{2}, z_{2}\right)=-2 \pi \sum_{k, n=1}^{\infty} \tilde{M}_{k n}^{(1)} \frac{a_{k} b_{n}}{\gamma_{k n}} \cos \left(a_{k} x_{2}\right) \cos \left(b_{n} y_{2}\right) \\
& \quad \times e^{-\gamma_{k n} z_{2}} \int_{-d_{1}}^{0} d z_{1} e^{\gamma_{k n} z_{1}}
\end{aligned}
$$

leading to the final expression

$$
\begin{aligned}
& H_{y}\left(x_{2}, y_{2}, z_{2}\right)=-2 \pi \sum_{k, n=1}^{\infty} \tilde{M}_{k n}^{(1)} \frac{a_{k} b_{n}}{\gamma_{k n}} e^{-\gamma_{k n} z_{2}}\left(1-e^{-\gamma_{k n} d_{1}}\right) \\
& \quad \times \cos \left(a_{k} x_{2}\right) \cos \left(b_{n} y_{2}\right) .
\end{aligned}
$$

In a similar way the component $H_{x}$ from Eq. (B1) can be written in the form

$$
\begin{aligned}
& H_{x}\left(x_{2}, y_{2}, z_{2}\right)=-\sum_{k, n=1}^{\infty} \tilde{M}_{k n}^{(1)} \int_{-\infty}^{\infty} d x_{1} \int_{-\infty}^{\infty} d y_{1} \int_{-d_{1}}^{0} d z_{1} \\
& \times\left\{\left(x_{2}-x_{1}\right) \frac{\partial}{\partial x_{2}} \frac{\sin \left(a_{k} x_{1}\right) \sin \left(b_{n} y_{1}\right)}{\left[\left(x_{2}-x_{1}\right)^{2}+\left(y_{2}-y_{1}\right)^{2}+\left(z_{2}-z_{1}\right)^{2}\right]^{3 / 2}}\right. \\
& \left.\quad+\frac{\sin \left(a_{k} x_{1}\right) \sin \left(b_{n} y_{1}\right)}{\left[\left(x_{2}-x_{1}\right)^{2}+\left(y_{2}-y_{1}\right)^{2}+\left(z_{2}-z_{1}\right)^{2}\right]^{3 / 2}}\right\} .
\end{aligned}
$$

Then we again introduce the new variables $u$ and $v$, set to zero the integrals of odd functions, calculate the derivative with respect to $x_{2}$ and introduce the derivative with respect to $a_{k}$ 
in order to use Eqs. ( $(\underline{\mathrm{A} 4})$ and (A5). These allow the following representation of Eq. (B8)$)$ :

$$
\begin{aligned}
& H_{x}\left(x_{2}, y_{2}, z_{2}\right)=-2 \pi \sum_{k, n=1}^{\infty} \tilde{M}_{k n}^{(1)} \sin \left(a_{k} x_{2}\right) \sin \left(b_{n} y_{2}\right) \\
& \times\left[-\frac{a_{k}^{2}}{\gamma_{k n}} \int_{-d_{1}}^{0} d z_{1} e^{-\gamma_{k n}\left(z_{2}-z_{1}\right)}+\int_{-d_{1}}^{0} \frac{d z_{1}}{z_{2}-z_{1}} e^{-\gamma_{k n}\left(z_{2}-z_{1}\right)}\right] .
\end{aligned}
$$

After integration and identical transformations one finally obtains

$$
\begin{aligned}
& H_{x}\left(x_{2}, y_{2}, z_{2}\right)=2 \pi \sum_{k, n=1}^{\infty} \tilde{M}_{k n}^{(1)} \sin \left(a_{k} x_{2}\right) \sin \left(b_{n} y_{2}\right) \\
& \times\left\{\frac{a_{k}^{2}}{\gamma_{k n}^{2}} e^{-\gamma_{k n} z_{2}}\left(1-e^{-\gamma_{k n} d_{1}}\right)+\operatorname{Ei}\left(-\gamma_{k n} z_{2}\right)-\operatorname{Ei}\left[-\gamma_{k n}\left(z_{2}+d_{1}\right)\right]\right\} .
\end{aligned}
$$

The magnetic energy between two parallel plates with in-plane magnetization is obtained from Eq. (22)

$$
E_{m}(a)=-\int_{0}^{L_{x}^{(1)}} d x_{2} \int_{0}^{L_{y}^{(1)}} d y_{2} \int_{a}^{a+d_{2}} d z_{2}\left(M_{x}^{(2)} H_{x}+M_{y}^{(2)} H_{y}\right) .
$$

Substituting Eqs. (37), (B7) and (B10) in Eq. (B11), one arrives at Eq. (38).

In the end we consider the case when the spontaneous magnetization is not equal to zero. We can again assume that the second film is situated above the center of the first and put $x_{2} \approx y_{2} \approx 0$. From symmetry considerations it also follows that $H_{y} \approx 0$. Then Eq. (19) written for the in-plane magnetization leads to

$$
\begin{gathered}
H_{x}\left(z_{2}\right)=\tilde{M}_{00}^{(1)} \int_{-L_{x}^{(1)} / 2}^{L_{x}^{(1)} / 2} d x_{1} \int_{-L_{y}^{(1)} / 2}^{L_{y}^{(1)} / 2} d y_{1} \int_{-d_{1}}^{0} d z_{1}\left\{\frac{3 x_{1}^{2}}{\left[x_{1}^{2}+y_{1}^{2}+\left(z_{2}-z_{1}\right)^{2}\right]^{5 / 2}}\right. \\
\left.-\frac{1}{\left[x_{1}^{2}+y_{1}^{2}+\left(z_{2}-z_{1}\right)^{2}\right]^{3 / 2}}\right\} .
\end{gathered}
$$

Using the identity

$$
\frac{3 x_{1}^{2}}{\left[x_{1}^{2}+y_{1}^{2}+\left(z_{2}-z_{1}\right)^{2}\right]^{5 / 2}}=-x_{1} \frac{\partial}{\partial x_{1}} \frac{1}{\left[x_{1}^{2}+y_{1}^{2}+\left(z_{2}-z_{1}\right)^{2}\right]^{3 / 2}},
$$

we calculate the integral with respect to $x_{1}$ and obtain

$$
H_{x}\left(z_{2}\right)=8 \tilde{M}_{00}^{(1)} L_{x}^{(1)} \int_{-L_{y}^{(1)} / 2}^{L_{y}^{(1)} / 2} d y_{1} \int_{-d_{1}}^{0} d z_{1} \frac{1}{\left[\left(L_{x}^{(1)}\right)^{2}+4 y_{1}^{2}+4\left(z_{2}-z_{1}\right)^{2}\right]^{3 / 2}} .
$$

Both integrations in Eq. (B14) can be easily performed with the result

$H_{x}\left(z_{2}\right)=4 \tilde{M}_{00}^{(1)}\left[\arctan \frac{2\left(z_{2}+d_{1}\right)}{\sqrt{\left(L_{x}^{(1)}\right)^{2}+\left(L_{y}^{(1)}\right)^{2}+4\left(z_{2}+d_{1}\right)^{2}}}-\arctan \frac{2 z_{2}}{\sqrt{\left(L_{x}^{(1)}\right)^{2}+\left(L_{y}^{(1)}\right)^{2}+4 z_{2}^{2}}}\right]$. 
Substituting Eq. (B15) in the following expression for the magnetic energy per unit area due to the spontaneous magnetization:

$$
E_{m s}(a)=-\tilde{M}_{x ; 00}^{(2)} \int_{a}^{a+d_{2}} d z_{2} H_{x}\left(z_{2}\right),
$$

one arrives at Eq. (41).

1 V. A. Parsegian, Van der Waals Forces: A Handbook for Biologists, Chemists, Engineers, and Physicists (Cambridge University Press, Cambridge, 2005).

2 H. B. G. Casimir, Proc. K. Ned. Akad. Wet. B 51, 793 (1948).

3 E. M. Lifshitz, Zh. Eksp. Teor. Fiz. 29, 94 (1955) [Sov. Phys. JETP 2, 73 (1956)].

4 M. Kardar and R. Golestanian, Rev. Mod. Phys. 71, 1233 (1999).

5 A. I. Volokitin and B. N. J. Persson, Rev. Mod. Phys. 79, 1291 (2007).

6 R. H. French, V. A. Parsegian, R. Podgornik, et al., Rev. Mod. Phys. 82, 1887 (2010).

7 V. M. Mostepanenko and N. N. Trunov, The Casimir Effect and its Applications (Clarendon, Oxford, 1997).

8 K. A. Milton, The Casimir Effect (World Scientific, Singapore, 2001).

9 M. Bordag, G. L. Klimchitskaya, U. Mohideen, and V. M. Mostepanenko, Advances in the Casimir Effect (Oxford University Press, Oxford, 2009).

10 G. L. Klimchitskaya, U. Mohideen, and V. M. Mostepanenko, Rev. Mod. Phys. 81, 1827 (2009).

11 A. W. Rodriguez, F. Capasso, and S. G. Johnson, Nature Photon. 5, 211 (2011).

12 G. L. Klimchitskaya, U. Mohideen, and V. M. Mostepanenko, Int. J. Mod. Phys. B 25, 171 (2011).

13 P. W. Selwood, Magnetochemistry (Interscience Publ., New York, 1956).

14 A. H. Morrish, The Physical Principles of Magnetism (J. Wiley, New York, 1965).

15 S. V. Vonsovskii, Magnetism (J. Wiley, New York, 1974).

16 J. H. Van Vleck, The Theory of Electric and Magnetic Susceptibilities (Oxford University Press, Oxford, 1932).

17 Yu. S. Barash and V. L. Ginzburg, Usp. Fiz. Nauk 116, 5 (1975) [Sov. Phys. Usp. 18, 305 (1975)].

18 P. Richmond and B. W. Ninham, J. Phys. C: Solid St. Phys. 4, 1988 (1971). 
19 S. Y. Buhmann, D.-G. Welsch, and T. Kampf, Phys. Rev. A 72, 032112 (2005).

20 M. S. Tomaš, Phys. Lett. A 342, 381 (2005).

21 S. J. Rahi, T. Emig, N. Graham, R. L. Jaffe, and M. Kardar, Phys. Rev. D 80, 085021 (2009).

22 O. Kenneth, I. Klich, A. Mann, and M. Revzen, Phys. Rev. Lett. 89, 033001 (2002).

23 D. Iannuzzi and F. Capasso, Phys. Rev. Lett. 91, 029101 (2003).

24 F. S. S. Rosa, D. A. R. Dalvit, and P. W. Milonni, Phys. Rev. A 78, 032117 (2008).

25 B. Geyer, G. L. Klimchitskaya, and V. M. Mostepanenko, Phys. Rev. B 81, 104101 (2010).

26 G. L. Klimchitskaya, B. Geyer, and V. M. Mostepanenko, Int. J. Mod. Phys. A 25, 2293 (2010).

27 N. Inui, Phys. Rev. A 84, 052505 (2011).

28 N. Inui, Phys. Rev. A 86, 022520 (2012).

29 G. Feinberg and J. Sucher, Phys. Rev. A 2, 2395 (1970).

30 R. S. Turtelli, R. Grössinger, and C. Kussbach, J. Appl. Phys. 83, 1581 (1998).

31 H. Safari, D.-G. Welsch, S. Y. Buhmann, and S. Scheel, Phys. Rev. A 78, 062901 (2008).

32 G. Bimonte, G. L. Klimchitskaya, and V. M. Mostepanenko, Phys. Rev. A 79, 042906 (2009).

33 A. A. Banishev, C.-C. Chang, G. L. Klimchitskaya, V. M. Mostepanenko, and U. Mohideen, Phys. Rev. B 85, 195422 (2012).

34 T. R. Albrecht, P. Grütter, D. Horne, and D. Rugar, J. Appl. Phys. 69, 668 (1991).

35 G. Jourdan. A. Lambrecht, F. Comin, and J. Chevrier, Europhys. Lett. 85, 31001 (2009).

36 J. Laurent, H. Sellier, A. Mosset, S. Huant, and J. Chevrier, Phys. Rev. B 85, 035426 (2012).

37 S. de Man, K. Heeck, R. J. Wijngaarden, and D. Iannuzzi, Phys. Rev. Lett. 103, 040402 (2009).

38 S. de Man, K. Heeck, and D. Iannuzzi, Phys. Rev. A 79, 024102 (2009).

39 F. J. Giessibl, Rev. Mod. Phys. 75, 949 (2003).

40 C.-C. Chang, A. A. Banishev, R. Castillo-Garza, G. L. Klimchitskaya, V. M. Mostepanenko, and U. Mohideen, Phys. Rev. B 85, 165443 (2012).

41 H. B. Chan, V. A. Aksyuk, R. N. Kleiman, D. J. Bishop, and F. Capasso, Science 291, 1941 (2001).

42 H. B. Chan, V. A. Aksyuk, R. N. Kleiman, D. J. Bishop, and F. Capasso, Phys. Rev. Lett. 87, 211801 (2001).

43 R. S. Decca, E. Fischbach, G. L. Klimchitskaya, D. E. Krause, D. López, and V. M. Mostepanenko, Phys. Rev. D 68, 116003 (2003).

44 R. S. Decca, D. López, E. Fischbach, G. L. Klimchitskaya, D. E. Krause, and V. M. Mostepa- 
nenko, Ann. Phys. (N.Y.) 318, 37 (2005).

45 R. S. Decca, D. López, E. Fischbach, G. L. Klimchitskaya, D. E. Krause, and V. M. Mostepanenko, Phys. Rev. D 75, 077101 (2007).

46 R. S. Decca, D. López, E. Fischbach, G. L. Klimchitskaya, D. E. Krause, and V. M. Mostepanenko, Eur. Phys. J. C 51, 963 (2007).

47 H. B. Chan, Y. Bao, J. Zou, R. A. Cirelli, F. Klemens, W. M. Mansfield and C. S. Pai, Phys. Rev. Lett. 101, 030401 (2008).

48 Y. Bao, R. Guérout, J. Lussange, A. Lambrecht, R. A. Cirelli, F. Klemens, W. M. Mansfield, C. S. Pai and H. B. Chan, Phys. Rev. Lett. 105, 250402 (2010).

49 A. A. Banishev, G. L. Klimchitskaya, V. M. Mostepanenko, and U. Mohideen, Phys. Rev. Lett. 10, 137401 (2013).

50 D. Rugar, H. J. Mamin, and P. Guethner, Appl. Phys. Lett. 55, 2588 (1989).

51 B. C. Stipe, H. J. Mamin, T. D. Stowe, T. W. Kenny, and D. Rugar, Phys. Rev. Lett. 87, 096801 (2001).

52 W. R. Smythe, Electrostatics and Electrodynamics (McGraw-Hill, New York, 1950).

53 F. Chen, U. Mohideen, G. L. Klimchitskaya, and V. M. Mostepanenko, Phys. Rev. A 74, 022103 (2006).

54 Handbook of Optical Constants of Solids, ed. E. D. Palik (Academic, New York, 1985).

55 A. O. Sushkov, W. J. Kim, D. A. R. Dalvit, and S. K. Lamoreaux, Nature Phys. 7, 230 (2011).

56 D. Garcia-Sanches, K. Y. Fong, H. Bhaskaran, S. Lamoreaux, and H. X. Tang, Phys. Rev. Lett. 109, $027202(2012)$.

57 V. B. Bezerra, G. L. Klimchitskaya, U. Mohideen, V. M. Mostepanenko, and C. Romero, Phys. Rev. B 83, 075417 (2011).

58 G. L. Klimchitskaya, M. Bordag, E. Fischbach, D. E. Krause, and V. M. Mostepanenko, Int. J. Mod. Phys. A 26, 3918 (2011).

59 G. L. Klimchitskaya, M. Bordag, and V. M. Mostepanenko, Int. J. Mod. Phys. A 27, 1260012 (2012).

60 M. Bordag, G. L. Klimchitskaya, and V. M. Mostepanenko, Phys. Rev. Lett. 109, 199701 (2012).

61 F. Chen, G. L. Klimchitskaya, V. M. Mostepanenko, and U. Mohideen, Optics Express 15, 4823 (2007).

62 F. Chen, G. L. Klimchitskaya, V. M. Mostepanenko, and U. Mohideen, Phys. Rev. B 76, 035338 
(2007).

63 J. M. Obrecht, R. J. Wild, M. Antezza, L. P. Pitaevskii, S. Stringari, and E. A. Cornell, Phys. Rev. Lett. 98, 063201 (2007).

64 G. L. Klimchitskaya and V. M. Mostepanenko, J. Phys. A: Math. Theor. 41, 312002 (2008).

65 C.-C. Chang, A. A. Banishev, G. L. Klimchitskaya, V. M. Mostepanenko, and U. Mohideen, Phys. Rev. Lett. 107, 090403 (2011).

66 A. A. Banishev, C.-C. Chang, R. Castillo-Garza, G. L. Klimchitskaya, V. M. Mostepanenko, and U. Mohideen, Phys. Rev. B 85, 045436 (2012).

67 V. B. Bezerra, G. L. Klimchitskaya, V. M. Mostepanenko, and C. Romero, Phys. Rev. A 69, $022119(2004)$.

68 B. Geyer, G. L. Klimchitskaya, and V. M. Mostepanenko, Phys. Rev. D 72, 085009 (2005).

69 G. L. Klimchitskaya, U. Mohideen, and V. M. Mostepanenko, J. Phys. A: Math. Theor. 40, 339 (2007).

70 K. A. Milton, J. Phys. A: Math. Gen. 37, R209 (2004).

71 I. Brevik, J. B. Aarseth, J. S. Høye, and K. A. Milton, Phys. Rev. E 71, 056101 (2005).

72 V. B. Bezerra, R. S. Decca, E. Fischbach, B. Geyer, G. L. Klimchitskaya, D. López, V. M. Mostepanenko, and C. Romero, Phys. Rev. E 73, 028101 (2006).

73 V. M. Mostepanenko and G. L. Klimchitskaya, Int. J. Mod. Phys. A 25, 2302 (2010).

74 M. A. Ordal, R. J. Bell, R. W. Alexander Jr., L. L. Long, and M. R. Querry, Appl. Opt. 24, 4493 (1985).

75 C. D. Fosco, F. C. Lombardo, and F. D. Mazzitelli, Phys. Rev. D 84, 105031 (2011).

76 G. Bimonte, T. Emig, R. L. Jaffe, and M. Kardar, Europhys. Lett. 97, 50001 (2012).

77 G. Bimonte, T. Emig, and M. Kardar, Appl. Phys. Lett. 100, 074110 (2012).

78 L. P. Teo, Phys. Rev. D 88, 045019 (2013).

79 R. S. Decca, E. Fischbach, G. L. Klimchitskaya, D. E. Krause, D. López, and V. M. Mostepanenko, Phys. Rev. D 79, 124021 (2009).

80 B. V. Derjaguin, Kolloid Zeitschrift. 69, 155 (1934).

81 S. Hameed, P. Talagala, R. Naik, L. E. Wenger, V. M. Naik, and R. Proksch, Phys. Rev. B 64, 184406 (2001).

82 W. H. Kraan and M. Th. Rekveldt, J. Magnetism \& Magn. Mat. 5, 247 (1977).

83 O. V. Snigirev, K. E. Andreev, A. M. Tishin, S. A. Gudoshnikov, and J. Bohr, Phys. Rev. B 
55, 14429 (1997).

84 M. Bordag, G. L. Klimchitskaya, and V. M. Mostepanenko, Int. J. Mod. Phys. A 10, 2661 (1995).

85 A. Wadas and P. Grütter, Phys. Rev. B 39, 12014 (1989).

86 D. Sarid, Scanning Force Microscopy. With Application to Electric, Magnetic and Atomic Forces (Oxford University Press, Oxford, 1994).

87 M. Mansuripur, IEEE Trans. Magn. 25, 3467 (1989).

88 I. S. Gradshtein and I. M. Ryzhik, Tables of Integrals, Series, and Products (Academic, New York, 1980).

89 D. Iannuzzi, I. Gelfand, M. Lisanti, and F. Capasso, In: K. A. Milton (ed.), Quantum Field Theory under the Influence of External Conditions (Rinton Press, Princeton, 2004), p.11.

90 C. C. Speake and C. Trenkel, Phys. Rev. Lett. 90, 160403 (2003).

91 A. A. Banishev, C.-C. Chang, R. Castillo-Garza, G. L. Klimchitskaya, V. M. Mostepanenko, and U. Mohideen, Int. J. Mod. Phys. A 27, 1260001 (2012).

92 G. Bimonte, Phys. Rev. A 83, 042109 (2011).

93 R. S. Decca, D. López, and E. Osquiguil, Int. J. Mod. Phys. A 25, 2223 (2010).

94 R. O. Behunin, F. Intravaia, D. A. R. Dalvit, P. A. Maia Neto, and S. Reynaud, Phys. Rev. A 85, $012504(2012)$.

95 R. S. Decca and G. Voisin, http://meetings.aps.org/Meeting/MAR13/Content/2502 

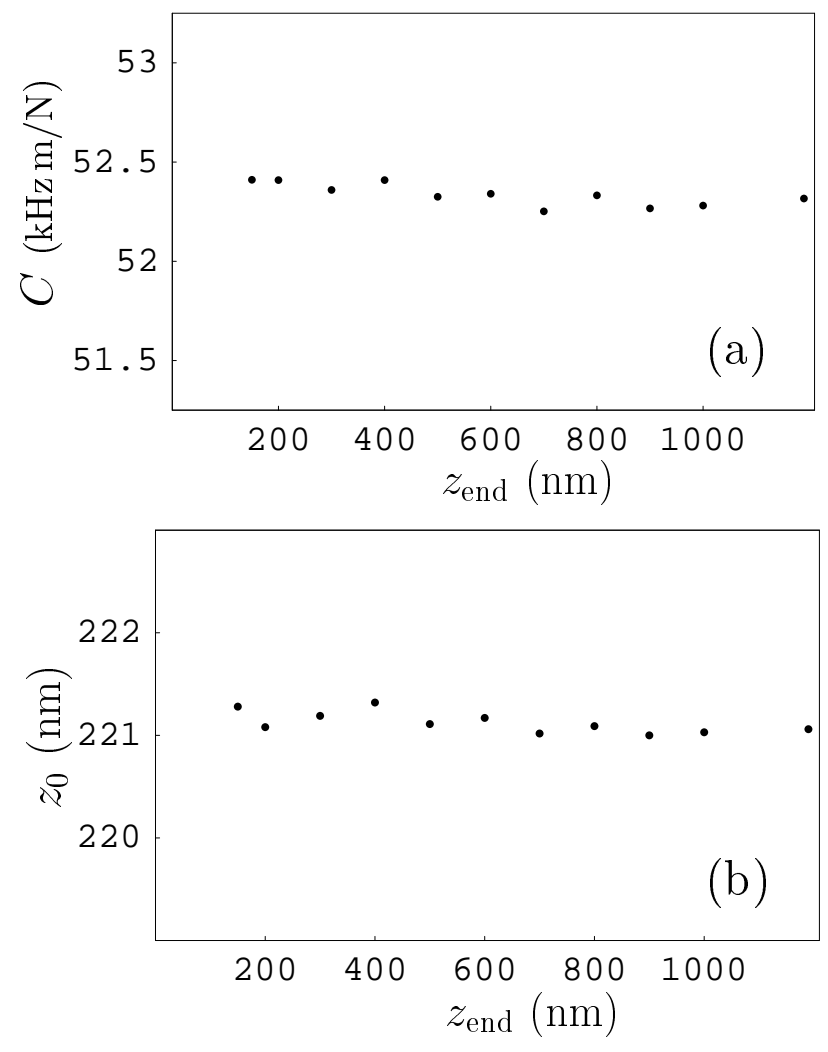

FIG. 1: (a) The coefficient $C$ in Eq. (10) and (b) the closest sphere-plate separation $z_{0}$ as functions of the end point of the fit. 


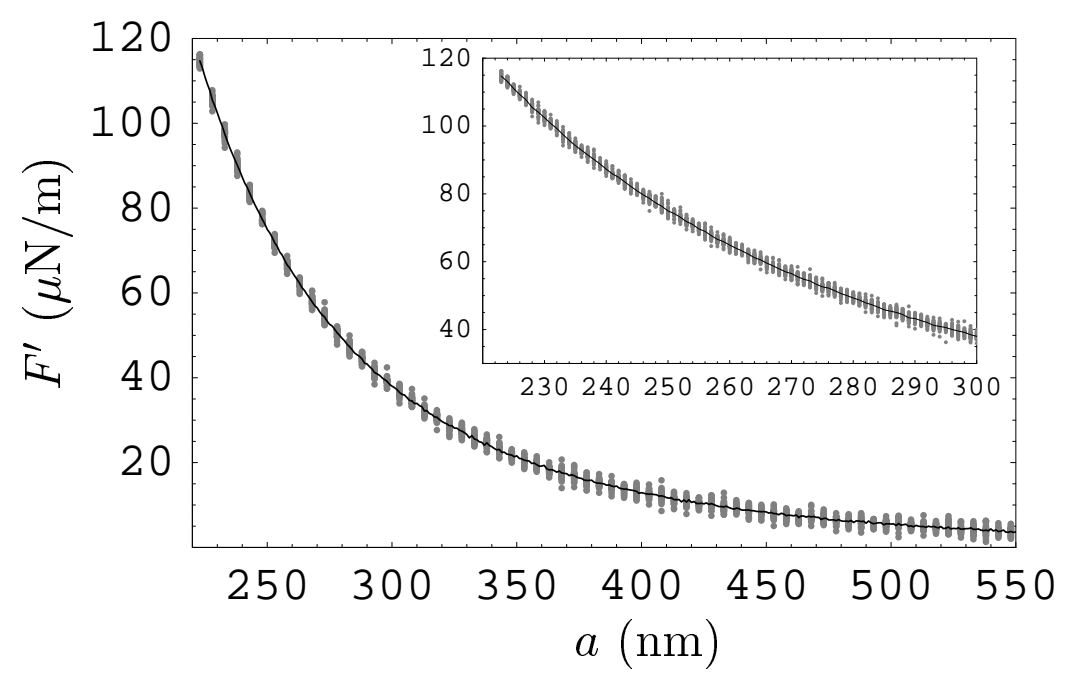

FIG. 2: All 33 data points for the gradient of the Casimir force between Ni surfaces are shown as dots with a step of $5 \mathrm{~nm}$ starting from a separation of $223 \mathrm{~nm}$. The mean values of the measured gradients are presented as the solid line. In the inset the same information is given with a step of $1 \mathrm{~nm}$ over a more narrow region.

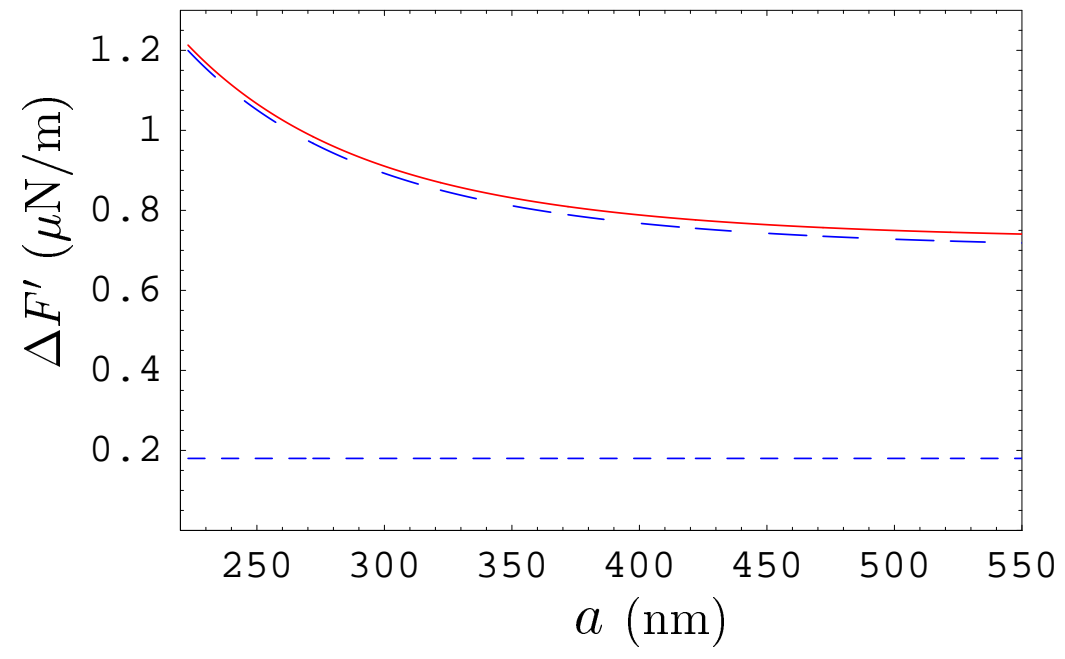

FIG. 3: (Color online) The random, $\Delta^{r} F^{\prime}$, systematic, $\Delta^{s} F^{\prime}$, and total, $\Delta^{t} F^{\prime}$, errors in the measured gradient of the Casimir force determined at a $67 \%$ confidence level are shown as functions of separation by the short-dashed, long-dashed, and solid lines, respectively. 


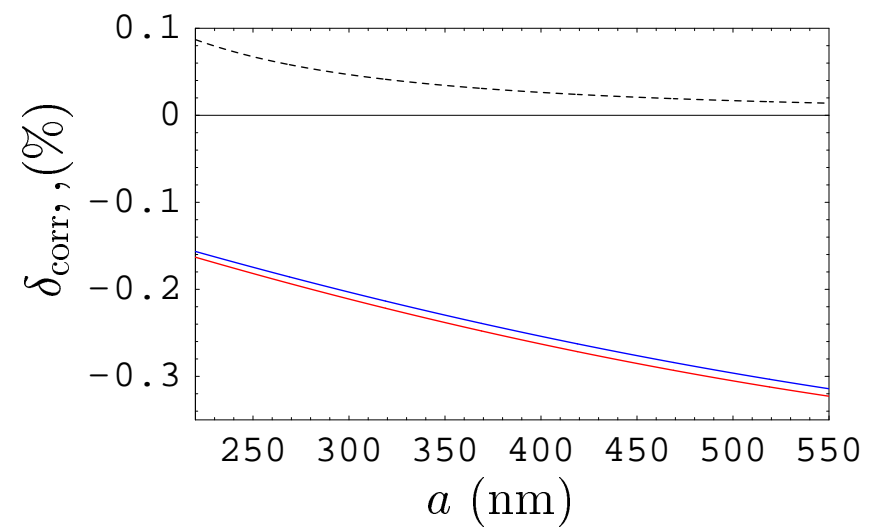

FIG. 4: (Color online) Corrections to the gradient of the Casimir force due to deviations from the PFA (upper and lower solid lines computed within the Drude and plasma model approaches, respectively) and due to the surface roughness (dashed line) as functions of separation.

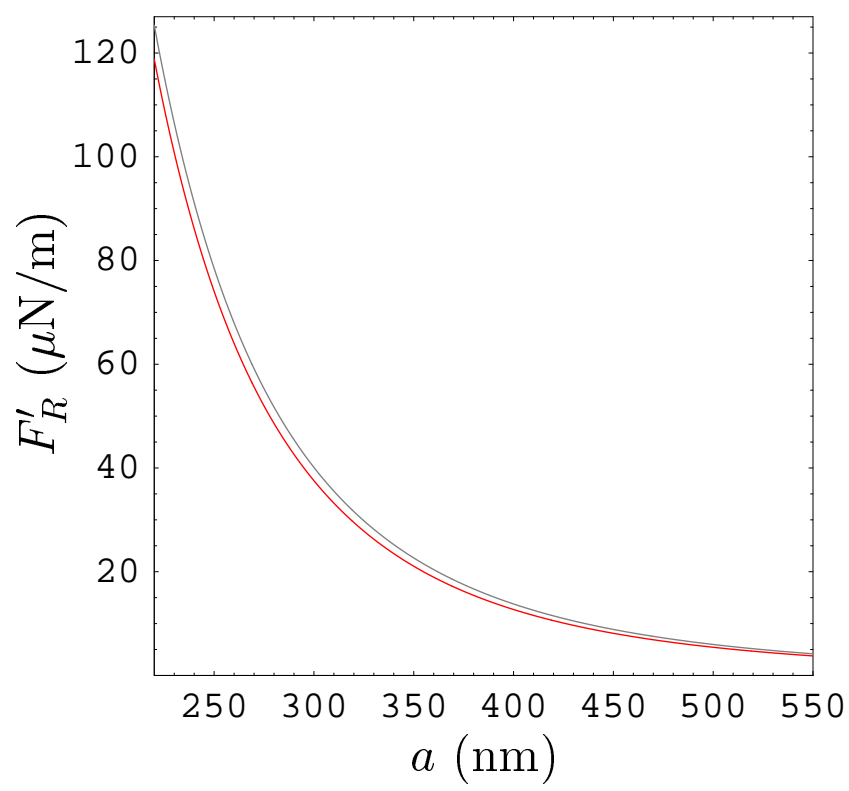

FIG. 5: (Color online) Theoretical predictions for the gradient of the Casimir force between Ni surfaces computed using the Drude and plasma model approaches (upper and lower lines, respectively) including corrections to the PFA and due to surface roughness as functions of separation. 

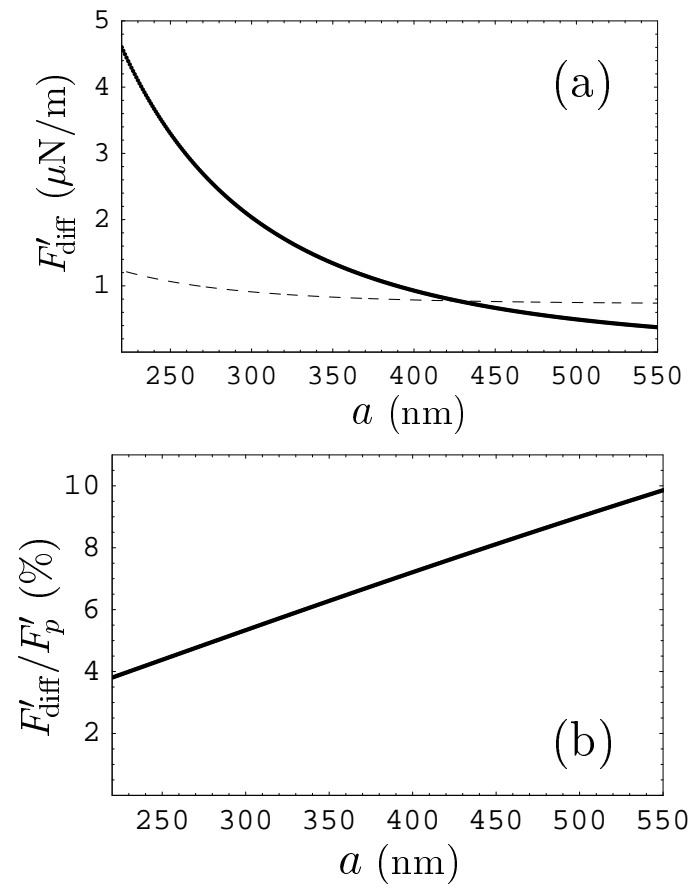

FIG. 6: (a) The difference of gradients of the Casimir force between Ni surfaces predicted within the Drude and plasma model approaches as a function of separation is shown by the solid line (the dashed line indicates the total experimental error determined at a $67 \%$ confidence level). (b) The relative difference of force gradients predicted within the Drude and plasma model approaches as a function of separation. 


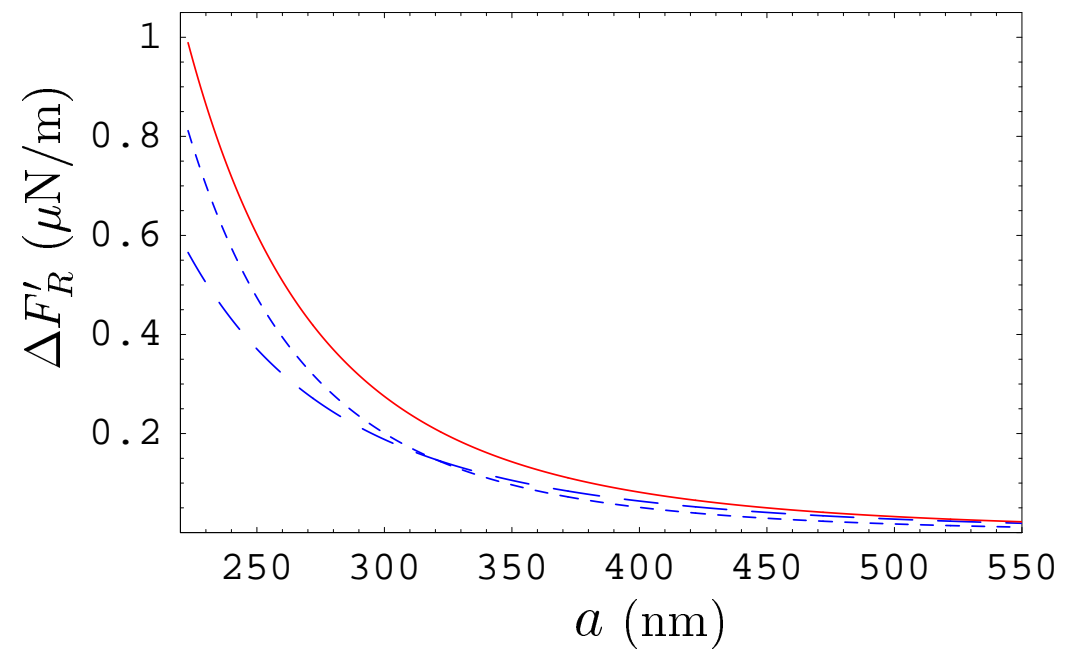

FIG. 7: (Color online) The errors in the theoretical gradient of the Casimir force $F_{R}^{\prime}\left(a_{i}\right)$ due to inaccuracy of the optical data of $\mathrm{Ni}, \Delta^{\mathrm{opt}} F_{R}^{\prime}$, due to the errors in measured separations $a_{i}, \Delta^{\mathrm{sep}} F_{R}^{\prime}$, and the total theoretical error, $\Delta^{t} F_{R}^{\prime}$, are shown as functions of separation by the long-dashed, short-dashed, and solid lines, respectively. 

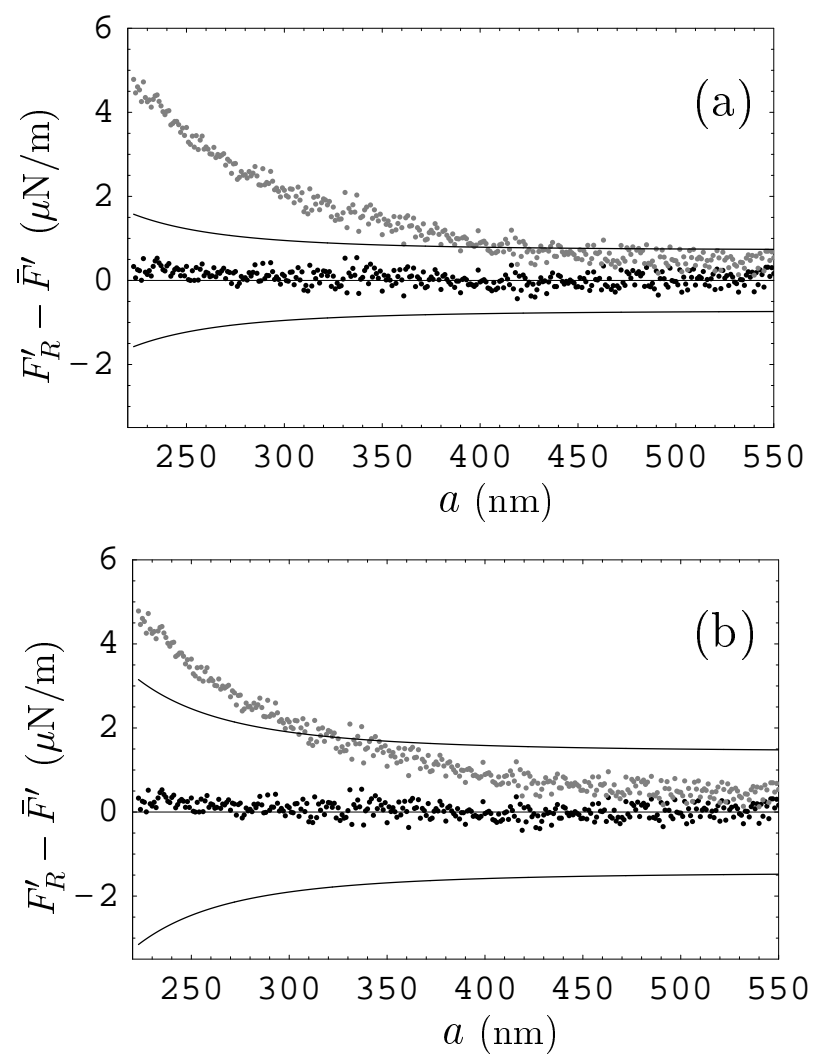

FIG. 8: Differences between the theoretical and mean experimental gradients of the Casimir force found at the experimental separations using the plasma and the Drude model approaches are shown by the black and gray dots, respectively. The solid lines indicate the borders of the (a) $67 \%$ and (b) $95 \%$ confidence intervals. 


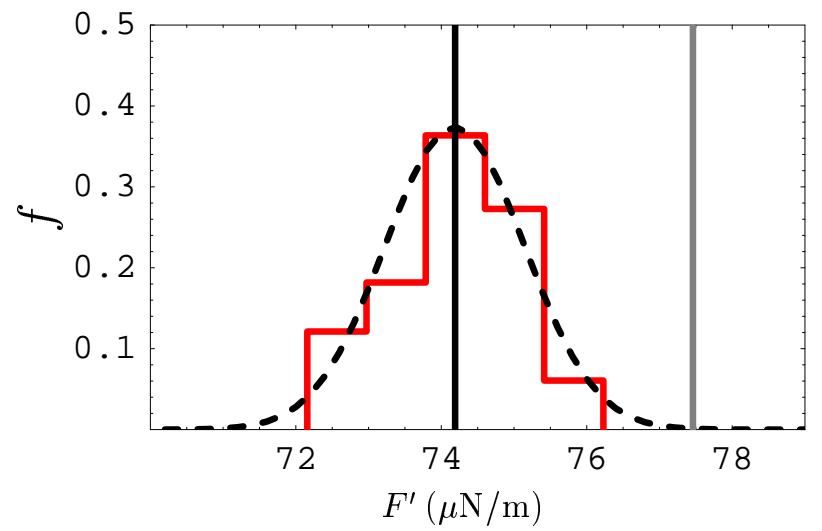

FIG. 9: (Color online) Histogram for the measured gradient of the Casimir force at the separation $a=250 \mathrm{~nm}$ (see text for details). The theoretical predictions of the plasma and Drude model approaches are shown by the black and gray vertical lines, respectively.

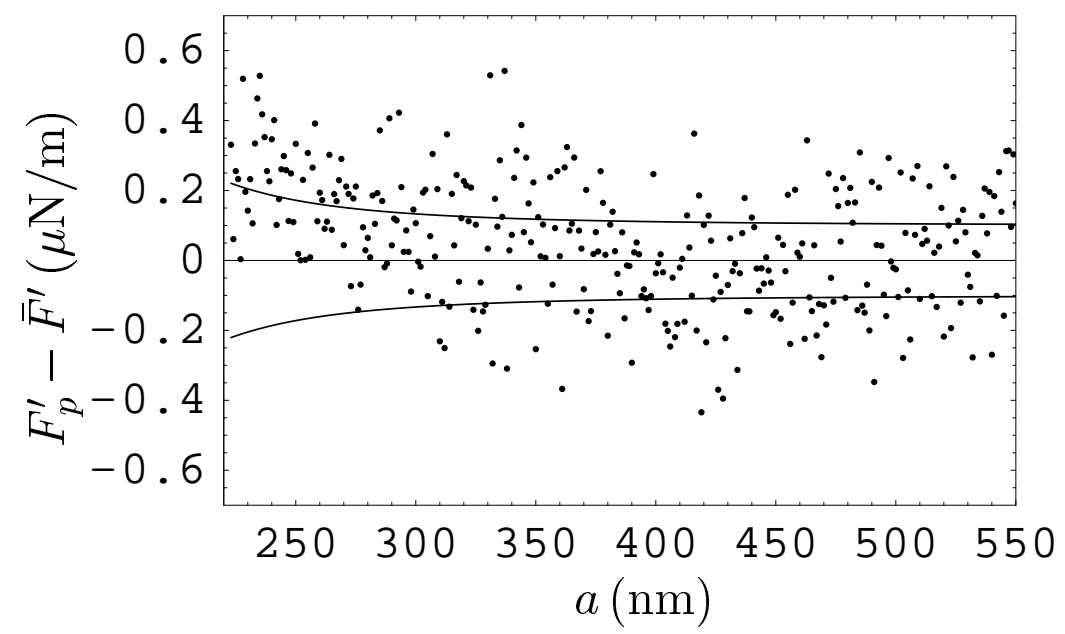

FIG. 10: Differences between the theoretical and mean experimental gradients of the Casimir force found at the experimental separations using the plasma model approach are shown by the black dots. The solid lines indicate the borders of the $10 \%$ confidence intervals. 

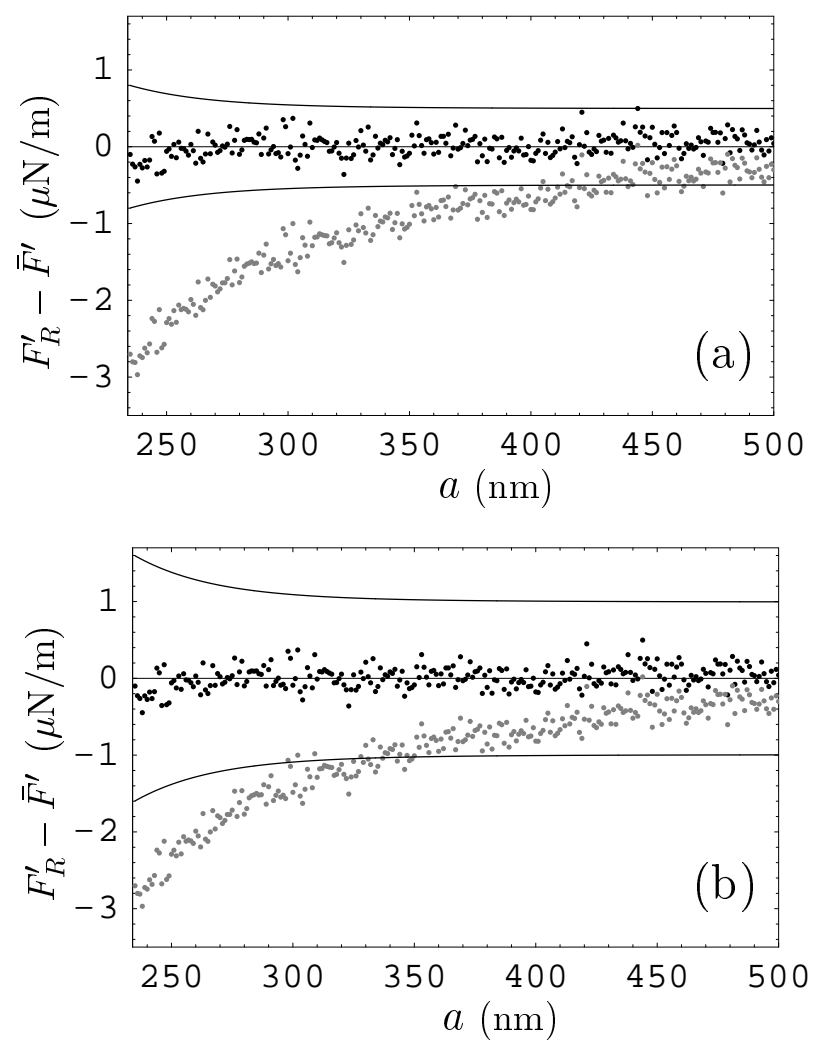

FIG. 11: Differences between the theoretical and mean experimental gradients of the Casimir force found at the experimental separations between a plate and a sphere both coated with Au using the plasma and the Drude model approaches are shown by the black and gray dots, respectively. The solid lines indicate the borders of the (a) $67 \%$ and (b) $95 \%$ confidence intervals. 

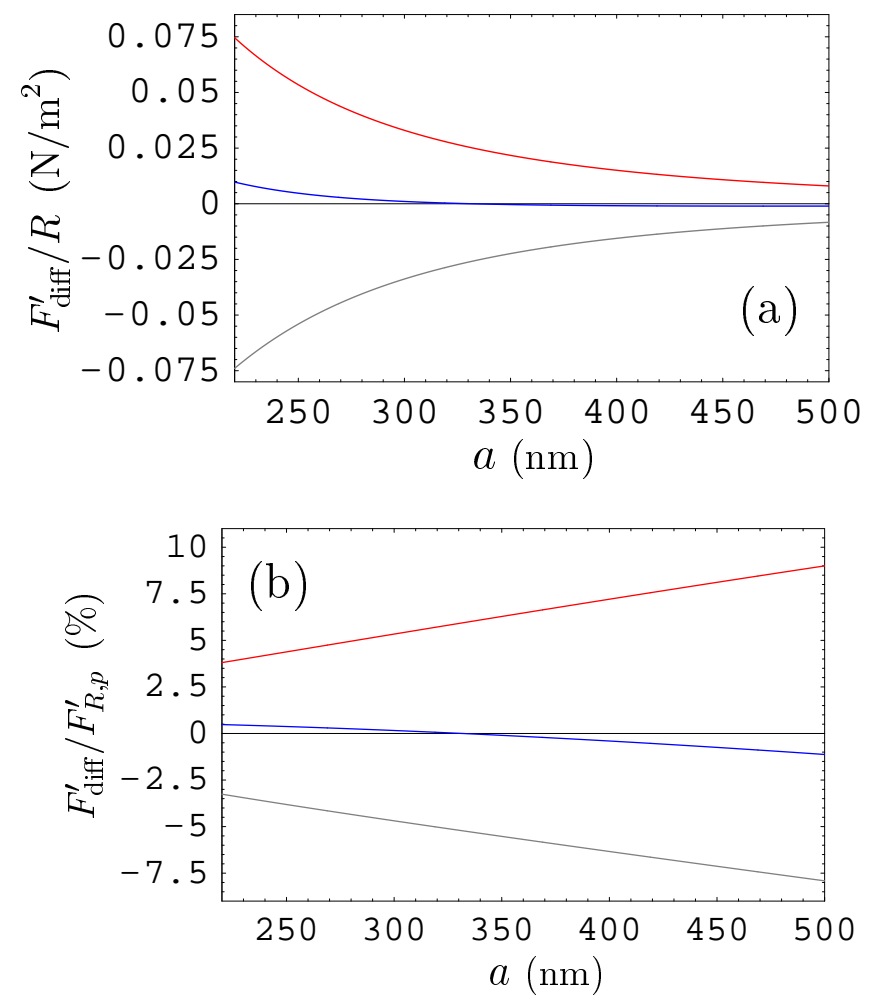

FIG. 12: (Color online) Difference of gradients of the Casimir force calculated using the Drude and plasma model approaches and normalized (a) for the respective sphere radii and (b) for the gradients of the Casimir force calculated using the plasma model approach are shown by the solid lines from top to bottom for experiments with $\mathrm{Ni}-\mathrm{Ni}, \mathrm{Ni}-\mathrm{Au}$ and $\mathrm{Au}-\mathrm{Au}$ test bodies, respectively. 

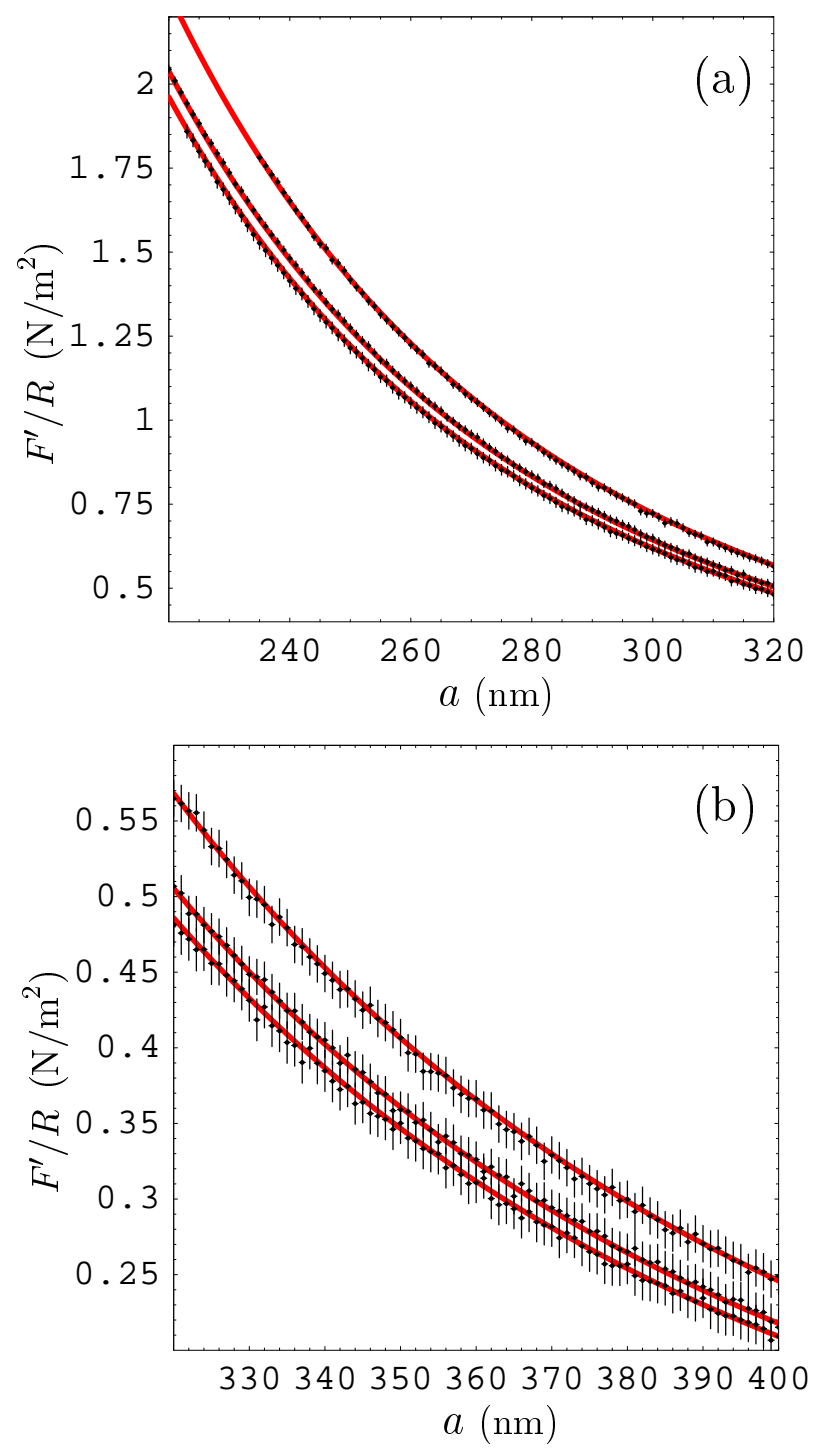

FIG. 13: (Color online) The measurement data for the mean gradients of the Casimir force normalized by sphere radii with the total experimental errors indicated as crosses and theoretical bands computed using the plasma model approach are shown from top to bottom for the experiments with $\mathrm{Au}-\mathrm{Au}, \mathrm{Au}-\mathrm{Ni}$ and $\mathrm{Ni}-\mathrm{Ni}$ surfaces over the separation region (a) from 220 to $320 \mathrm{~nm}$ and (b) from 320 to $400 \mathrm{~nm}$. 

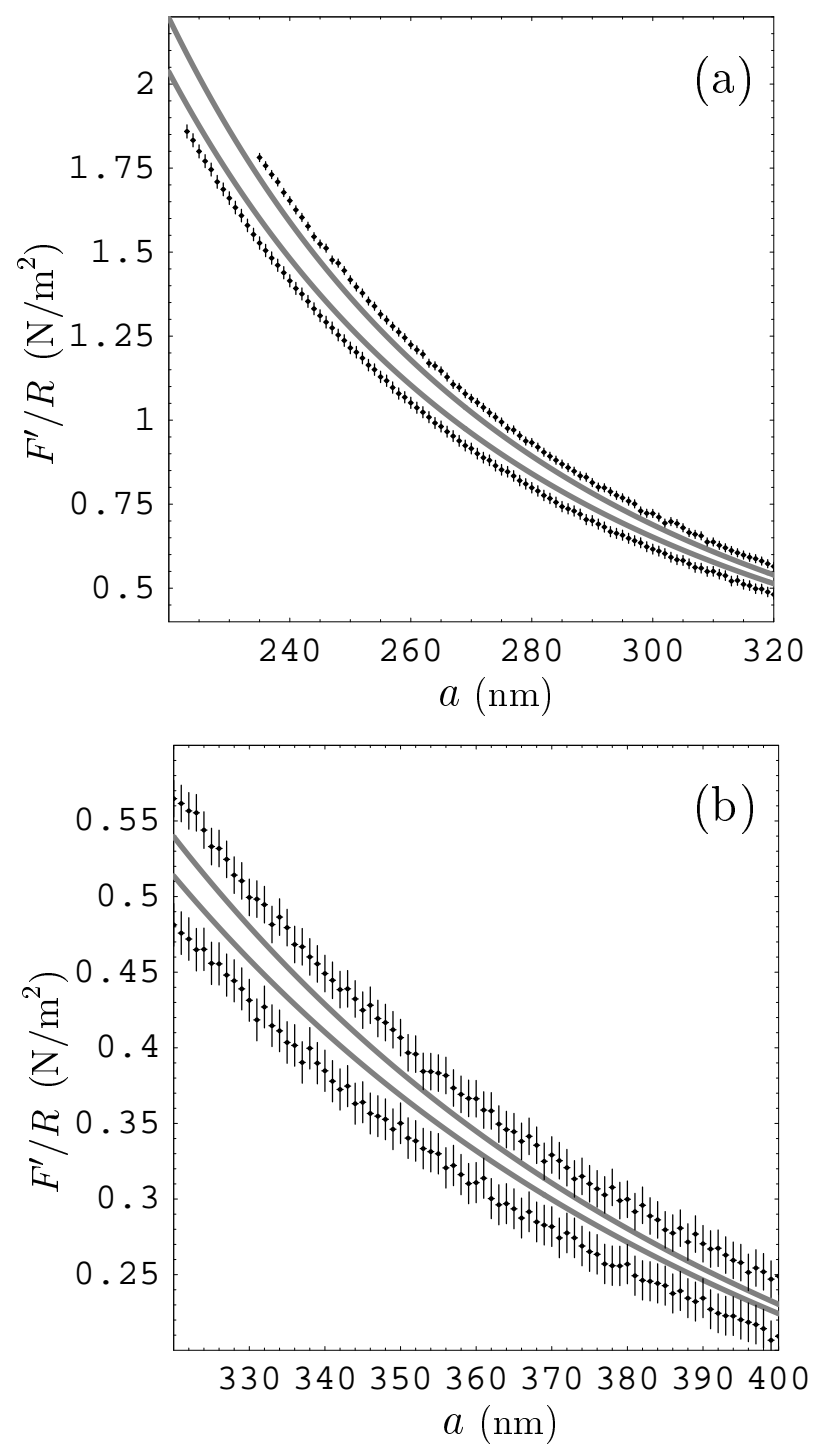

FIG. 14: The measurement data for mean gradients of the Casimir force normalized by sphere radii with total experimental errors indicated as crosses and the theoretical bands computed using the Drude model approach are shown from top to bottom for the experiments with $\mathrm{Au}-\mathrm{Au}$ and Ni-Ni surfaces over the separation region (a) from 220 to $320 \mathrm{~nm}$ and (b) from 320 to $400 \mathrm{~nm}$. 